\title{
Collective Choice in Dynamic Public Good Provision: Real versus Formal Authority*
}

\author{
T. Renee Bowen ${ }^{\dagger}$ \\ George Georgiadis ${ }^{\ddagger}$ \\ Nicolas S. Lambert ${ }^{\S}$
}

December 18, 2015

\begin{abstract}
Two heterogeneous agents exert effort over time to complete a project and collectively decide its scope. A larger scope requires greater cumulative effort and delivers higher benefits upon completion. To study the scope under collective choice, we derive the agents' preferences over scopes. The efficient agent prefers a smaller scope, and preferences are time-inconsistent: as the project progresses, the efficient agent's preferred scope shrinks, whereas the inefficient agent's preferred scope expands. In equilibrium without commitment, the efficient agent obtains his ideal project scope with either agent as dictator and under unanimity. In this sense, the efficient agent always has real authority.
\end{abstract}

JEL codes: C73, H41, D70, D78

Keywords: public goods, collective choice, real authority

${ }^{*}$ We thank Eduardo Azevedo, Marco Battaglini, Alessandro Bonatti, Steve Coate, Jakša Cvitanic̀, Jon Eguia, Mitchell Hoffman, Navin Kartik, Matias Iaryczower, Roger Lagunoff, Tom Palfrey, Patrick Rey, Andy Skrzypacz, Leeat Yariv, and participants at Stanford University, Cornell Public and Microeconomics Workshop, the CRETE 2015, INFORMS 2015, SAET 2015, Yale Political Economy 2015 conferences, the Econometric Society World Congress 2015, and Stony Brook Game Theory Festival Political Economy Workshop 2015 for helpful comments and suggestions.

${ }^{\dagger}$ Stanford Graduate School of Business, Stanford, CA 94305, U.S.A.; trbowen@stanford.edu.

${ }^{\ddagger}$ Kellogg School of Management, Northwestern University, Evanston, IL 60208, U.S.A; ggeorgiadis@kellogg.northwestern.edu.

${ }^{\S}$ Stanford Graduate School of Business, Stanford, CA 94305, U.S.A.; nlambert@stanford.edu. 


\section{Introduction}

In many economic settings, agents must collectively decide the goal or scope of a public project. A greater scope reflects a more ambitious project, which requires more effort from each agent but yields a greater reward upon completion. Such collective decisions are common among countries seeking to cooperate on a project. As an example, the International Space Station (ISS) was a collaboration among the United States, Russia, the European Union, Japan, and Canada that cost approximately $\$ 150$ billon. The Asian Highway Network, running about eighty-seven thousand miles and costing over $\$ 25$ billion, is a collaboration among thirty-two Asian countries, the United Nations (UN), and other entities to facilitate greater trade throughout the region. In both examples, the projects took several decades to implement, an agreement was signed by all countries, and this agreement determined the project scope. ${ }^{1}$ Other examples include infrastructure projects jointly undertaken between states or municipalities. The Gordie Howe International Bridge, for instance, is a joint project between the Michigan Department of Transportation in the United States and the Ministry of Transportation of Ontario in Canada. It started in 2015 with estimated costs of more than $\$ 2$ billion (see Associated Press, 2015). In these settings, if the agents' preferences over the project scope are aligned, then the natural choice for the project scope is the mutually agreed upon ideal, and there will be little debate. Yet, it is common to find disagreement about when and at what stage to complete a public project. For example, the process of identifying roads to be included in the Asian Highway Network began in the late 1950s, but it was not until the 1990s that the majority of the work began, owing to the endorsement of the UN (see Yamamoto et al., 2003). The World Trade Organization's (WTO's) Doha Round began in 2001 and has (infamously) yet to be concluded fifteen years later. The delay owes, in part, to differences between member countries over which industries the agreement should cover and to what extent (see Bhagwati and Sutherland, 2011). ${ }^{2}$ Central to many of these conflicts is the asymmetry between participants - often large contributors versus small contributors. In this paper we investigate how the agents' cost of effort and their

\footnotetext{
${ }^{1}$ Other notable multi-country collaborations include the International Thermonuclear Experimental Reactor (ITER) under construction in France, and the Joint European Torus (JET) in the United Kingdom.

${ }^{2}$ Other explanations are plausible for delays in public projects, including, unanticipated costs, or natural or socioeconomic disasters (such as wars). In this paper, timing of the project is entirely due to incentives to exert effort, which are in turn driven by the choice of project scope.
} 
stake in the project affect their incentives to contribute and, ultimately, their real authority to influence the project scope under various collective choice institutions.

We focus on public projects with three key features. First, progress on the project is gradual, and hence the problem is dynamic in nature. Second, the agents' stake in the project, that is, the fraction of the project benefit that each agent receives upon completion, remains fixed once the project has been started. Third, the project generates a payoff predominantly upon reaching the goal. Thus, the scope of the project is a crucial determinant of not only the magnitude of the payoffs and effort, but also their timing. These features capture the main interrelated features of a public project - time, cost and scope. They are often referred to as the traditional triple constraint in the project management literature (see, for example, Dobson, 2004).

The features we consider also appear in settings beyond public projects. Many new business ventures require costly effort before payoffs can be realized. Indeed, there is often dissent on when a joint project is ready to be monetized through the launch of the product or sale of the company, for example. Academics working on a joint research project must exert voluntary effort over time, and the reward is largely realized after submission and publication of the findings. In both settings, agents will agree at some point in time on the scope of the project. Does the venture seek a blockbuster product or something that may have a quicker (if smaller) payoff? Do the coauthors target highly regarded general interest journals or work towards a more specific field journal? The analysis is well suited to these settings, but we maintain the focus on public projects.

A decision about a project's scope can be made at any time, with or without the ability to commit. As an example, it is common for the scope of a public infrastructure project to change throughout its development, a phenomenon often known as "scope creep." In such cases, the parties cannot commit to not renegotiate. In other settings, such as with an entrepreneurial venture, legally binding contracts can often be enforced. An agreement can then be made at any time during the project and parties can commit to it without the possibility of subsequent renegotiation. Importantly, the ability to commit is considered a part of the economic environment and is not a choice of the agents. The possibility for change in the project scope without commitment versus no change with commitment and the influence on authority is considered.

Formal authority is distinguished by the fact that it can be enforced by institutions outside of the agents' control, and it grants the holder the ability to complete the 
project and realize payoffs. That is, the agent with formal authority can unilaterally "pull the plug" and in this sense is the dictator. In the examples of the ISS and the Asian Highway Network, each country must sign a formal agreement for the project to enter into force (see Yakovenko, 1999; Yamamoto et al., 2003). In these examples, the scope of the project cannot be decided without the consent of all parties: the collective choice institution is unanimity, and we say that no single agent has formal authority. An agreement may also designate a single party with the right to complete the project, such as when one party has a controlling share of an entrepeneurial joint venture. In this setting, the controlling share endows the party with formal authority to sell the project and collect payoffs. The shares of the project in the entrepreneurial venture are the agents' stakes.

By contrast, real authority is not enforceable, but rather derived from the agents' endowed attributes. The attributes we consider in this paper are the agents' effort costs and stake in the project. Other attributes, such as endowed information, may confer real authority, as in the seminal work of Aghion and Tirole (1997). Although the model we present is substantially different from that of Aghion and Tirole (1997), our interpretations of real and formal authority are quite similar. Real authority is equivalently thought of as real control. In the public-project examples previously given, it may be inferred that the value of the contribution is the sole source of real authority, but in this paper we explore an alternate perspective - each agent's cost of effort relative to his stake in the project determines his incentives to exert effort (and hence incur costs), which in turn, determines real authority. An agent with no incentive to exert effort can credibly stop making contributions to the project, hence determining the project completion state. We ask if the influence of the largest contributor to the joint project (for example, the United States) may be induced by its productivity relative to its partner countries and its stake in the project.

In the examples of the ISS, the Asian Highway Network and the WTO, the larger countries contribute the most and are understood to have the greatest influence, although each agreement is formally governed by unanimity. The US is reported to have contributed $\$ 58$ billion of the $\$ 150$ billion to the ISS, and some estimate that the total contribution of the US is closer to $\$ 100$ billion (see, Plumer, 2014). Our paper sheds light on the question of why large countries dominate international decisions when the collective choice institution is formally unanimity.

The modeling approach we take is based on the dynamic public good provision 
framework of Marx and Matthews (2000). In practice, the project scope may encompass multiple dimensions. However, in this framework we make the simplifying assumption that the project scope is its size and is, thus, single-dimensional. It is well established in this setting that free-riding occurs when agents must make voluntary contributions. Basic comparative statics (e.g., the effect of changes in effort costs, discount rates, scope, etc.) are well understood when agents are symmetric. However, little is known about this problem when agents are heterogeneous. In many settings of interest, including several previously described, multiple heterogeneous agents must make the collective decision. We begin by studying a simple two-agent model. The agent with the lower effort cost per unit of benefit is the efficient agent, and the agent with the higher effort cost per unit of benefit is inefficient. We take the standard approach even further by establishing the agents' endogenous preferences over the project scope. Preferences are, thus, determined by the agents' per-unit cost of effort and stake in the project. Once preferences for the project scope are established, we study the choice of project scope under two collective choice institutions - dictatorship and unanimity - considering that agents may or may not have the power to commit to the decision.

The solution concept we use is Markov perfect equilibrium, as is standard in this literature. These equilibria require minimal coordination and memory and are, in this sense, simple. Where multiple equilibria exist, we refine the set of equilibria to the surplus maximizing ones. ${ }^{3}$

Our first set of results concern the setting in which the project scope and stakes are exogenously fixed. We show that the efficient agent exerts more effort than the inefficient agent at every stage of the project and, moreover, gets a lower discounted payoff (normalized by his project stake). The reason is that, in spite of having a lower per-unit cost of effort, the efficient agent is penalized by the magnitude of the effort he exerts to the extent that his normalized payoff is lower. In a similar setting with completely symmetric agents, it has been established that the agents' efforts increase closer to the end of the project because the discounting of the future payoff plays a smaller role (see Georgiadis, 2015). We show that the same is true with asymmetric agents, and we further show that the efficient agent's effort increases at a faster rate than that of the inefficient agent, and thus the efficient agent bears a greater share of

\footnotetext{
${ }^{3}$ The main results are robust to considering Pareto-dominant equilibria, but these are not unique in all cases.
} 
the remaining total project costs the closer the project is to completion.

We use our results about the agents' effort choices for a fixed project scope to derive their endogenous preferences over the project scope. A lower normalized payoff for the efficient agent means that at every stage of the project the efficient agent prefers a smaller project scope than does the inefficient agent. Furthermore, we show that the scope of the project that the efficient agent wants decreases as the project progresses, and the reverse is true for the inefficient agent. This is because the efficient agent's share of the remaining project cost is not only higher than the inefficient agent's, but also increases as the project progresses. The agents' preferences for the project scope are thus time-inconsistent and divergent.

Next, we study the choice of project scope when it can be selected at any time by collective choice, and we consider the implications for real and formal authority. We model formal authority as the ability to determine the state at which the project ends and rewards are collected. Formal authority is therefore determined by the collective choice institution. The agent who is dictator is said to have formal authority, and if unanimity is the collective choice institution, then neither agent has formal authority. We say that an agent has real authority if the project scope is the agent's ideal at the moment it is decided. In the setting we study it is not always the case that an agent with the ability to end the project unilaterally (i.e. the dictator) does so at a state he considers ideal.

We summarize the results with and without commitment. With commitment, we show that the project scope is decided at the start of the project in equilibrium under any institution. When either agent is dictator, he achieves his ex-ante ideal project scope. With unanimity and commitment, the project scope lies between the agents' ex-ante ideal scopes and neither agent has real authority. Real and formal authority are thus equivalent with commitment. Without commitment, the project scope is not decided until completion in equilibrium. The efficient agent as dictator achieves his ideal project scope at completion, so he has real and formal authority in this case. However, when the inefficient agent is dictator, the equilibrium project scope lies between the agents' ideal scopes. That is, at completion, the efficient agent wishes to stop the project immediately, but the inefficient agent would prefer to continue, so the efficient agent has real authority. The same is true under unanimity. Thus, without commitment, the efficient agent retains control, and formal authority is not equivalent to real authority. 
Our final set of results concern social welfare. We consider the choice of a social planner who seeks to maximize total surplus with her choice of project scope but is unable to coerce the agents to exert effort and thus takes as given the inefficiency due to free-riding. When the efficient agent is dictator, the equilibrium project scope is too small relative to the social planner's, with or without commitment. The reason is that he retains real authority in both cases, and his ideal project scope does not internalize the inefficient agent's higher dynamic payoff. If the inefficient agent is dictator, then the equilibrium project scope maximizes surplus without commitment. The intuition with commitment and the inefficient agent as dictator is the reverse of the intuition for the efficient agent - the inefficient agent has real authority and chooses a project scope that is too large. Without commitment, the inefficient agent does not have real authority, and the equilibrium project scope is the efficient agent's ideal at completion and also coincides with the social planner's ideal. Only with unanimity is the social planner's project scope part of an equilibrium with or without commitment, because both agents' payoffs can be internalized by the collective choice institution. With unanimity and no commitment, the equilibrium project scope is the social planner's ideal, yet the efficient agent retains real authority. This is because at the time of completion, the efficient agent wishes to stop immediately, whereas the inefficient agent would rather work towards a bigger project. This may explain the prevalence of unanimity as a collective choice institution in international organizations and may reconcile this with the seemingly outsized influence of larger and more efficient countries. ${ }^{4}$

The dominance of unanimity is robust to the inclusion of transfers, endogenizing project shares, and considering uncertainty. Such transfers are feasible if agents are not credit-constrained ex-ante. Unlimited transfers allow the agents to achieve the social planner's project scope under all institutions, and if the agents can choose the stakes (or shares) of the project ex-ante, simulations show that the efficient agent is always allocated a higher share than the inefficient agent. With the efficient agent as dictator, the share awarded to him is naturally the largest. Simulations also show that the main results hold with uncertainty. ${ }^{5}$ Unanimity surplus-dominates dictatorship in

\footnotetext{
${ }^{4}$ Efficiency may be measured by labor productivity, as an example. See Bureau of Labor Statistics (2011).

${ }^{5}$ The models with uncertainty and endogenous choice of project shares in the voluntary contribution game with heterogeneous agents that we study is analytically intractable, so we obtain results numerically. All other results in the paper are obtained analytically.
} 
all cases.

Our interest in real and formal authority relates to a mature academic literature studying the source of authority and power. Indeed, modern sociology attributes the three classifications of authority - traditional, charismatic, and legal-rational - to the pioneering work of Weber (1958). Weber (1958) was largely concerned with the determinants of legitimacy, and thus these three sources of authority can be thought of as sources of formal authority in our vernacular. In economics, the study of formal and informal authority also has a rich tradition, including the influential work of Aghion and Tirole (1997) and more recent contributions by Callander (2008), Callander and Harstad (2015), Hirsch and Shotts (2015), and Akerlof (2015). Unlike this paper, these authors focus on the role of information in determining real authority. Others have studied the link between institutions and power. Pfeffer (1981) and Williamson (1996), among others, consider theories of power and authority in organizations without formal models. Acemoglu and Robinson (2006b,a) and Acemoglu and Robinson (2008) consider the distinction between de jure political power and de facto political power. The source of de jure power is the formal political institution (such as dictatorship or democracy), and the source of de facto power is described as emerging "from the ability to engage in collective action, or use brute force or other channels such as lobbying or bribery" (Acemoglu and Robinson, 2006a). Loosely speaking, formal authority is the analog of de jure power in our setting, and real authority is the analog of de facto power. In these papers, de facto power is determined in equilibrium through investment and collective action, and the source is attributed to various forces outside the model. This is because the source of de facto power is extremely complicated in the political context. In contrast, we are able to endogenously attribute the source of real authority under different collective choice institutions to the cost of agents' effort in our simpler setting of a public project. We thus contribute to the literature on authority by providing an efficiency theory of real authority.

This paper joins a large political economy literature studying collective decisions when the agents' preferences are heterogeneous, including the seminal work of Romer and Rosenthal (1979). More recently, this literature has turned its attention to the dynamics of collective decision making, including papers by Baron (1996), Dixit et al. (2000), Battaglini and Coate (2008), Strulovici (2010), Diermeier and Fong (2011), Besley and Persson (2011) and Bowen et al. (2014). Other papers, for example, Lizzeri and Persico (2001), have looked at alternative collective choice institutions. Our 
paper joins this literature by studying the collective choice of agents deciding the scope of a long-term public project, and compares the outcomes under two different institutions - dictatorship and unanimity.

Our theory is closely related to numerous papers that take up the problem of agents providing voluntary contributions to a public good over time, including classic contributions by Levhari and Mirman (1980) and Fershtman and Nitzan (1991). Similarly to our approach, Admati and Perry (1991), Marx and Matthews (2000), Compte and Jehiel (2004), Yildirim (2006), Georgiadis et al. (2014), Georgiadis (2015), and Cvitanić and Georgiadis (2015) consider the case of public good provision when the benefit is received predominantly at completion. With the exception of Cvitanić and Georgiadis (2015), these papers consider symmetric agents, whereas we consider asymmetric agents. None of these papers considers collective choice of project scope, which is the focus of our analysis. Bonatti and Rantakari (forthcoming) consider collective choice in a public good game, but in their setting each agent exerts effort on an independent project, and the collective choice is made to adopt one of the projects at completion. In our setting, by contrast, agents work on a single collective project, decisions are made over project scope, and they can be made at any time during the project. Battaglini et al. (2014) consider a public good that delivers flow benefits and does not have a completion date, in contrast to our setting. This literature has been predominantly concerned with incentives to free ride. We contribute to it by considering agents' preferences over the project scope, the endogenous choice of the terminal state, and the implications for real and formal authority.

The application to public projects without the ability to commit relates to a large number of papers studying international agreements. Several of these study environmental agreements (for example, Nordhaus, 2015; Battaglini and Harstad, forthcoming) and trade agreements (see Maggi, 2014). ${ }^{6}$ To our knowledge, this literature has not examined the dynamic selection of project scope (or goals) in these agreements with asymmetric agents or identified the source of authority. Our theory sheds light on the dominance of large countries in many trade and environmental agreements in spite of a formal institution of unanimity.

The remainder of the paper is organized as follows. In Section 2 we present the

\footnotetext{
${ }^{6}$ Bagwell and Staiger (2002) discuss the economics of trade agreements in depth. Others look at various aspects of specific trade agreements, such as flexibility or forbearance in a non-binding agreement, (see, for example, Beshkar and Bond, 2010; Bowen, 2013).
} 
model of two agents deciding the scope of a public project. Section 3 characterizes the equilibrium of the game with an exogenous project scope to lay the foundation for the collective choice analysis. In this section we also provide the agents' ideal project scopes, and the social planner's benchmark results. In Section 4 we endogenize the project scope and examine the outcome under two collective choice institutionsdictatorship and unanimity - and present our main results about real and formal authority under each collective choice institution. In Section 5 we discuss the role of transfers and endogenous shares in enhancing the efficiency properties of the collective choice institutions. In this section we also demonstrate the robustness of the results to an environment with uncertainty. We conclude in Section 6.

\section{Model}

We present a stylized model of two heterogeneous agents $i \in\{1,2\}$ deciding the scope of a public project $Q \geq 0$. Time is continuous and indexed by $t \in[0, \infty)$. A project of scope $Q$ requires voluntary effort from the agents over time to be completed. Let $a_{i t} \geq 0$ be agent $i$ 's instantaneous effort level at time $t$, which induces flow cost $c_{i}\left(a_{i t}\right)=\gamma_{i} a_{i t}^{2} / 2$ for agent $i$. Agents are risk-neutral and discount time at common rate $r>0$.

Let $q_{t}$ denote the cumulative effort (or progress on the project) up to time $t$, which we call the project state. The project starts at initial state $q_{0}=0$ and evolves according to

$$
d q_{t}=\left(a_{1 t}+a_{2 t}\right) d t
$$

It is completed when the state reaches the chosen scope $Q \cdot{ }^{7}$ The project yields no payoff while it is in progress, but upon completion, it yields a payoff $\alpha_{i} Q$ to agent $i$, where $\alpha_{i} \in \mathbb{R}_{+}$is agent $i$ 's stake in the project. Agent $i$ 's project stake therefore captures all of the expected benefit from the project. For example, in the case of a public infrastructure project, this may include reduced traffic, cleaner water, greater opportunities for scientific discovery, and greater opportunities for domestic production. ${ }^{8}$

\footnotetext{
${ }^{7}$ For the main analysis, we present a deterministic baseline model. We discuss the extension to uncertainty in Section 5.2.

${ }^{8}$ If we impose the added restrictions that $\alpha_{1}+\alpha_{2}=1$, the project stake can be alternatively thought of as the project share. This interpretation is appropriate for the case of an entrepreneurial
} 
The project scope $Q$ is decided by collective choice at any time $t \geq 0$, i.e., at the start of the project, after some progress has been made, or at completion. The set of decisions available to each agent will depend on the collective choice institution. The collective choice institution is either dictatorship or unanimity. Under dictatorship, if agent $i$ is the dictator, then agent $i$ 's decision will be a choice of project scope $\theta_{i t} \in\left[q_{t}, \infty\right) \cup\{-1\}$. By convention, we let $\theta_{i t}=-1$ if no decision is made by agent $i$ as dictator. If agent $i$ is the dictator, then agent $j$ has no decision to make. Under unanimity, if agent $i$ is the proposer, then agent $i$ makes a proposal for the project scope $\theta_{i t} \in\left[q_{t}, \infty\right) \cup\{-1\}$, where, as before, $\theta_{i t}=-1$ is interpreted as no proposal. The other agent $j$ as the responder must make a decision to either agree or disagree, captured by $Y_{j t} \in\{0,1\}$, where $Y_{j t}=1$ if agent $j$ agrees to a proposal made at time $t$.

For each institution we consider two cases, with and without commitment. In the case with commitment, if a decision has been made, then agents are not allowed to reverse the decision, that is, agents are committed to the decided project scope. In the case without commitment, agents may revise their decision at any time, that is, agents are not committed to any decided project scope. In both cases, the project cannot be completed until a project scope is announced and imposed (in the case of dictatorship) or agreed upon (in the case of unanimity).

In the case of commitment and agent $i$ as dictator, if $T$ is the first time at which $\theta_{i T} \neq-1$, then $Q=\theta_{i T}$. Under commitment, the decision about the project scope may be thought of as signing a binding contract. Note that progress can be made on the project before and/or after such a contract is signed. If agent $i$ is the proposer under unanimity and with commitment, then $Q=\theta_{i T}$, where $T$ is the first time at which $\theta_{i T} \neq-1$ and $Y_{j T}=1$.

In the case of no commitment, we can focus on strategies in which $\theta_{i t}$ takes only values in $\left\{q_{t},-1\right\}$ for all $t \geq 0 .{ }^{9}$ If agent $i$ is the dictator, then $Q=\theta_{i t}$ if $\theta_{i t} \neq-1$. If agent $i$ is the proposer and unanimity is required, then $Q=\theta_{i t}$ if $Y_{j t}=1$ and $\theta_{i t} \neq-1$. The case of no commitment can be thought of as an environment in which there is no contract, or in which contracts are not enforceable, as is true with many international

venture, and the results we present can be applied. We wish to allow for the case of a pure public good, i.e., $\alpha_{1}=\alpha_{2}=1$, and we maintain the interpretation that $\alpha_{i}$ is agent $i$ 's project stake. The sum $\alpha_{1}+\alpha_{2}$ thus reflects the publicness of the good. Agents' stakes, of course, may be correlated, may vary through time, and project benefits may not be a linear function of the project scope. To begin our exploration of collective choice we make the simplifying assumptions that these stakes are independent, fixed through time, and the project benefit is the product of the scope and stake.

${ }^{9}$ This restriction is without loss of generality, as we explain in Section 4. 
agreements.

All information is common knowledge. Given an arbitrary set of effort paths $\left\{a_{1 s}, a_{2 s}\right\}_{s \geq t}$ and project scope $Q$, agent $i$ 's discounted payoff at time $t$ satisfies

$$
J_{i t}=e^{-r(\tau-t)} \alpha_{i} Q-\int_{t}^{\tau} e^{-r(s-t)} \frac{\gamma_{i}}{2} a_{i s}^{2} d s,
$$

where $\tau$ denotes the completion time of the project (and $\tau=\infty$ if the project is never completed).

By convention, we assume that the agents are ordered such that $\frac{\gamma_{1}}{\alpha_{1}} \leq \frac{\gamma_{2}}{\alpha_{2}}$. Intuitively, this means that agent 1 is relatively more efficient than agent 2 , in that his marginal cost of effort relative to his stake in the project is less than that of agent 2. That is, the ratio $\frac{\gamma_{i}}{\alpha_{i}}$ measures agent $i$ 's cost of effort per unit of project benefit. We say that agent 1 is efficient and agent 2 is inefficient. ${ }^{10}$

\section{Foundations}

In this section, we lay the foundations for the collective choice analysis. We begin by considering the case in which the project scope $Q$ is specified exogenously at the outset of the game and characterize the stationary Markov perfect equilibria (hereafter MPE) of this game. ${ }^{11}$ We then derive each agent's preferences over the project scope $Q$ given the MPE payoffs induced by a choice of $Q$. Last, we characterize the social planner's benchmark. In Section 4, we consider the case in which the agents decide the project scope via collective choice.

\subsection{Markov perfect equilibrium with exogenous project scope}

We characterize the unique MPE of the game in which each agent observes the current project state $q$ and chooses his effort level to maximize his discounted payoff while anticipating the other agents' effort choices for a fixed project scope $Q$.

In an MPE, each agent's discounted continuation payoff and effort level are a function of the project state $q$. We denote these by $J_{i}(q)$ and $a_{i}(q)$, respectively. Using

\footnotetext{
${ }^{10}$ The efficient agent is equivalently the high stakes agent, since efficiency is defined relative to project stake. In particular, our setting allows for both agents to have the same marginal cost of effort, but different project stakes.

${ }^{11}$ As is standard in this literature, we focus on Markov perfect equilibria. These equilibria require minimal coordination between the agents, and in this sense they are simple. The simplicity of Markov equilibria make them naturally focal in the collective choice setting.
} 
standard arguments (for example, Kamien and Schwartz, 2012), if the functions $J_{i}(q)$, $i=1,2$ are continuously differentiable, then they satisfy the Hamilton-Jacobi-Bellman (hereafter HJB) equation

$$
r J_{i}(q)=\max _{\widehat{a}_{i} \geq 0}\left\{-\frac{\gamma_{i}}{2} \widehat{a}_{i}^{2}+\left(\widehat{a}_{i}+a_{j}(q)\right) J_{i}^{\prime}(q)\right\},
$$

subject to the boundary condition

$$
J_{i}(Q)=\alpha_{i} Q
$$

where $a_{j}$ is agent $i$ 's conjecture for the effort levels chosen by agent $j \neq i$.

The right side of (1) is maximized when $\widehat{a}_{i}=\max \left\{0, J_{i}^{\prime}(q) / \gamma_{i}\right\}$. Intuitively, this means that an agent either does not put in any effort or, by the first-order condition, chooses his effort level such that the marginal cost of effort is equal to the marginal benefit associated with bringing the project closer to completion at every moment. In any equilibrium we have $J_{i}^{\prime}(q) \geq 0$ for all $i$ and $q$, that is, each agent is better off the closer the project is to completion. ${ }^{12}$ By substituting each agent's first-order condition into (1), it follows that each agent $i$ 's discounted payoff function satisfies

$$
r J_{i}(q)=\frac{\left[J_{i}^{\prime}(q)\right]^{2}}{2 \gamma_{i}}+\frac{1}{\gamma_{j}} J_{i}^{\prime}(q) J_{j}^{\prime}(q),
$$

subject to the boundary condition (2), where $j$ denotes the agent other than $i .^{13}$

By noting that each agent's problem is concave, and thus the first-order condition is necessary and sufficient for a maximum, it follows that every MPE is characterized by the system of ordinary differential equations (ODEs) defined by (3) subject to (2). We focus on MPEs such that $J_{1}$ and $J_{2}$ are continuous and satisfy piecewise differentiability. We refer to such MPEs as well-behaved. The following proposition characterizes the well-behaved MPE of this game.

Proposition 1. For any project scope $Q$, there exists a unique well-behaved MPE. Moreover for any project scope $Q$, exactly one of two cases can occur.

1. The MPE is project-completing: both agents exert effort at all states up to completion and complete the project. Then, $J_{i}(q)>0, J_{i}^{\prime}(q)>0$, and $a_{i}^{\prime}(q)>0$ for both agents $i$ and all states $q \geq 0$.

\footnotetext{
${ }^{12}$ See the proof of Proposition 1.

${ }^{13}$ This system of ODEs can be normalized by letting $\widetilde{J}_{i}(q)=\frac{J_{i}(q)}{\gamma_{i}}$. This becomes strategically equivalent to a game in which $\gamma_{1}=\gamma_{2}=1$, and agent $i$ receives $\frac{\alpha_{i}}{\gamma_{i}} Q$ upon completion of the project.
} 
2. The MPE is not project-completing: agents do not start working on the project, and both agents make zero payoffs.

Finally, if $Q$ is sufficiently small, then case (1) applies, while if $Q$ is sufficiently large, then case (2) applies.

All proofs are relegated to the Appendix.

Proposition 1 characterizes the unique MPE given a possible value of $Q$. Given a value of $Q$, either the project is never undertaken and payoffs are zero, or efforts are strictly positive and the project is completed. Note that in any project-completing MPE, each agent increases his effort as the project progresses towards completion, i.e., $a_{i}^{\prime}(q)>0$ for all $i$. Intuitively, because the agents discount time and they are rewarded only upon completion, their incentives are stronger the closer the project is to completion. An implication of this result is that efforts are strategic complements across time in this model. This is because by raising his effort, an agent brings the project closer to completion, thus inducing the other agent to raise his future efforts. ${ }^{14}$

It is straightforward to show that if agents are symmetric (i.e., if $\frac{\gamma_{1}}{\alpha_{1}}=\frac{\gamma_{2}}{\alpha_{2}}$ ), then in the unique project-completing MPE, each agent $i$ 's discounted payoff and effort function satisfies

$$
J_{i}(q)=\frac{r \gamma_{i}(q-C)^{2}}{6} \quad \text { and } \quad a_{i}(q)=\frac{r(q-C)}{3}
$$

respectively, where $C=Q-\sqrt{\frac{6 \alpha_{i} Q}{r \gamma_{i}}} .{ }^{15}$ This implies that when the agents are symmetric, they exert the same amount of effort, and the agent with the lower cost of effort attains a lower payoff. While the solution to the system of ODEs given by (3) subject to (2) can be found with relative ease in the case of symmetric agents, no closed-form solution can be obtained for the case of asymmetric agents. Nonetheless, we are able to derive important properties of the solution. The following proposition compares the equilibrium effort levels and payoffs of the two agents.

Proposition 2. Suppose that $\frac{\gamma_{1}}{\alpha_{1}}<\frac{\gamma_{2}}{\alpha_{2}}$. In any project-completing MPE:

1. Agent 1 exerts higher effort than agent 2 in every state, and agent 1 's effort increases at a greater rate than agent 2's. That is, $a_{1}(q) \geq a_{2}(q)$ and $a_{1}^{\prime}(q) \geq$ $a_{2}^{\prime}(q)$ for all $q \geq 0$.

\footnotetext{
${ }^{14}$ Strategic complementarity has been shown with symmetric agents by Kessing (2007) and with asymmetric agents by Cvitanić and Georgiadis (2015).

${ }^{15}$ This result follows from Georgiadis et al. (2014).
} 
2. Agent 1 obtains a lower discounted payoff normalized by project stake than agent 2. That is, $\frac{J_{1}(q)}{\alpha_{1}} \leq \frac{J_{2}(q)}{\alpha_{2}}$ for all $q \geq 0$.

Suppose instead that $\frac{\gamma_{1}}{\alpha_{1}}=\frac{\gamma_{2}}{\alpha_{2}}$. In any project-completing MPE, $a_{1}(q)=a_{2}(q)$ and $\frac{J_{1}(q)}{\alpha_{1}}=\frac{J_{2}(q)}{\alpha_{2}}$ for all $q \geq 0$.

It is intuitive that the more efficient agent always exerts higher effort than the less efficient agent. What is perhaps surprising is the result that the more efficient agent obtains a lower discounted payoff (normalized by his stake) than the other agent. This is because the more efficient agent not only works harder than the other agent, but he also incurs a higher total discounted cost of effort (normalized by his stake).

\subsection{Preferences over project scope}

\section{Agents working jointly}

It is necessary to understand the agents' preferences over project scopes to obtain the equilibrium project scope under collective choice. We characterize each agent's optimal project scope without institutional restrictions. That is, we determine the $Q$ that maximizes each agent's discounted payoff given the current state $q$ and assuming that both agents follow the MPE characterized in Proposition 1 for the project scope Q. Based on Proposition 1, the agents will choose a project scope such that the project is completed in equilibrium and each agent obtains a strictly positive payoff. Thus, the agents will not choose a project scope such that neither agent chooses to put in effort, and so we focus on project scopes with strictly positive effort choices, i.e., those for which the MPE is project-completing.

To make the dependence on the project scope explicit, we now let $J_{i}(q ; Q)$ denote agent $i$ 's value function at project state $q$ when the project scope is $Q$. An example of the function $J_{i}(q ; Q)$ is given in Figure 1 below.

Let $Q_{i}(q)$ denote agent $i$ 's ideal project scope when the state of the project is $q$. That is,

$$
Q_{i}(q)=\arg \max _{Q \geq q}\left\{J_{i}(q ; Q)\right\} .
$$

Note that for each agent $i$ there exists a unique value of $q$, which we denote $\bar{Q}_{i}$, such that agent $i$ is indifferent between terminating the project immediately, and terminating the project an instant later. ${ }^{16}$ The remaining results of the paper hold

\footnotetext{
${ }^{16}$ We provide the values of $\bar{Q}_{i}$ in Lemma 6 in Section A.1 of the Appendix.
} 


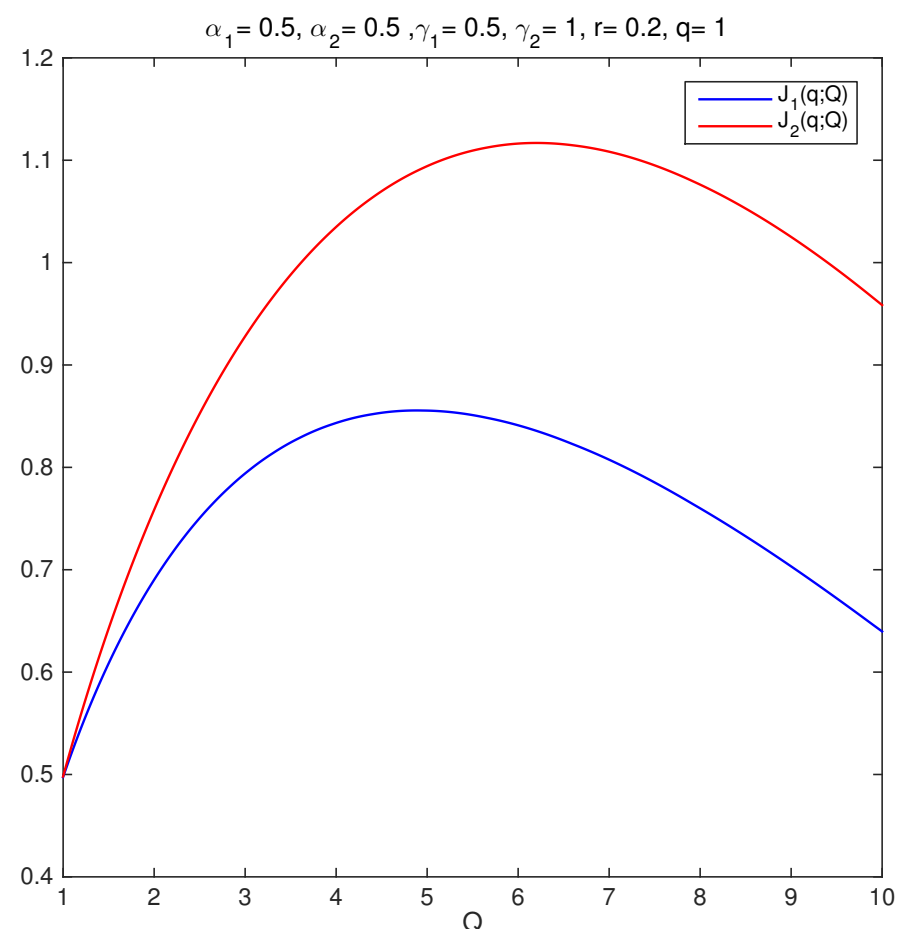

Figure 1: $J_{i}(q ; Q)$ as a function of $Q$

under the condition that $Q \mapsto J_{i}(q ; Q)$ is strictly concave on $\left[q, \bar{Q}_{2}\right]$ and reaches its maximum in that interval. ${ }^{17}$

The following proposition establishes properties of an agent's optimal project scope.

Proposition 3. Consider agent $i$ 's optimal project scope $Q$ when both agents choose their effort strategies based on $Q$.

1. If the agents are symmetric, i.e., $\frac{\gamma_{1}}{\alpha_{1}}=\frac{\gamma_{2}}{\alpha_{2}}$, then for all states $q$, their ideal project scope is the same and given by $Q_{1}(q)=Q_{2}(q)=\frac{3 \alpha_{2}}{2 \gamma_{2} r}$.

2. If the agents are asymmetric, i.e., $\frac{\gamma_{1}}{\alpha_{1}}<\frac{\gamma_{2}}{\alpha_{2}}$, then

(a) The efficient agent prefers a strictly smaller project scope than the inefficient agent at all states up to $\bar{Q}_{2}$, i.e., $Q_{1}(q)<Q_{2}(q)$ for all $q<\bar{Q}_{2}$.

(b) The efficient agent's ideal scope is strictly decreasing in the project state up to $\bar{Q}_{1}$, while the inefficient agent's scope is strictly increasing for all q, i.e.,

\footnotetext{
${ }^{17}$ While we do not provide a formal proof, numerous numerical simulations suggest that this condition holds.
} 


$$
Q_{1}^{\prime}(q)<0 \text { for all } q \leq \bar{Q}_{1} \text { and } Q_{2}^{\prime}(q)>0 \text { for all } q
$$

(c) Agent i's ideal is to complete the project immediately at all states greater than $\bar{Q}_{i}$, i.e., $Q_{i}(q)=q$ for all $q \geq \bar{Q}_{i}$.

Proposition 3.1 asserts that when the agents are symmetric, they have identical preferences over project scope, and these preferences are time-consistent.

Proposition 3.2 is illustrated in Figure 2 with the values $\bar{Q}_{1}$ and $\bar{Q}_{2}$ indicated. It characterizes each agent's ideal project scope when the agents are asymmetric. Part (a) asserts that the more efficient agent always prefers a strictly smaller project scope than the less efficient agent for $q<\bar{Q}_{2} \cdot{ }^{18}$ Note that each agent trades off the bigger gross payoff from a project with a larger scope and the cost associated with having to exert more effort and wait longer until the project is completed. Moreover, agent 1 not only always works harder than agent 2 , but at every moment, his discounted total cost remaining to complete the project normalized by his stake (along the equilibrium path) is larger than that of agent $2 .{ }^{19}$ Therefore, it is intuitive that agent 1 prefers a smaller project scope than agent 2 .

Proposition 3.2(b) shows that both agents are time-inconsistent with respect to their preferred project scope: as the project progresses, agent 1's optimal project scope becomes smaller, whereas agent 2 would like to choose an ever larger project scope. To see the intuition behind this result, recall that $a_{1}^{\prime}(q) \geq a_{2}^{\prime}(q)>0$ for all $q$; that is, both agents increase their effort with progress, but the rate of increase is greater for agent 1 than it is for agent 2. This implies that for a given project scope, the closer the project is to completion, the larger is the share of the remaining effort carried out by agent 1 . Therefore, agent 1's optimal project scope decreases. The converse holds for agent 2 , and as a result, his preferred project scope becomes larger as the project progresses.

Proposition 3.2(c) gives agent $i$ 's ideal project scope when the state $q$ is larger than $\bar{Q}_{i}$ for each agent $i$. Recall that $\bar{Q}_{i}$ is the project scope such that agent $i$ is indifferent between stopping immediately (when $q=\bar{Q}_{i}$ ) and continuing one instant longer. This is the value of the state at which $Q_{i}(q)$ hits the $45^{\circ}$ line. For states above $\bar{Q}_{i}$, agent

\footnotetext{
${ }^{18}$ The agents' ideal project scopes are equal for $q \geq \bar{Q}_{2}$ by Proposition 3.2 part (c), so the efficient agent's ideal is always weakly lower.

${ }^{19}$ Formally and as implied by Proposition 2.2, for every $t \in[0, \tau)$, we have $\frac{\gamma_{1}}{\alpha_{1}} \int_{t}^{\tau} e^{-r t} \frac{a_{1}^{2}\left(q_{t} ; Q\right)}{2} d t>$ $\frac{\gamma_{2}}{\alpha_{2}} \int_{t}^{\tau} e^{-r t} \frac{a_{2}^{2}\left(q_{t} ; Q\right)}{2} d t$ along the equilibrium path of the project.
} 
$i$ prefers to stop immediately. Agent $i$ 's ideal project scope is therefore the current state of the project for all states above $\bar{Q}_{i}$.

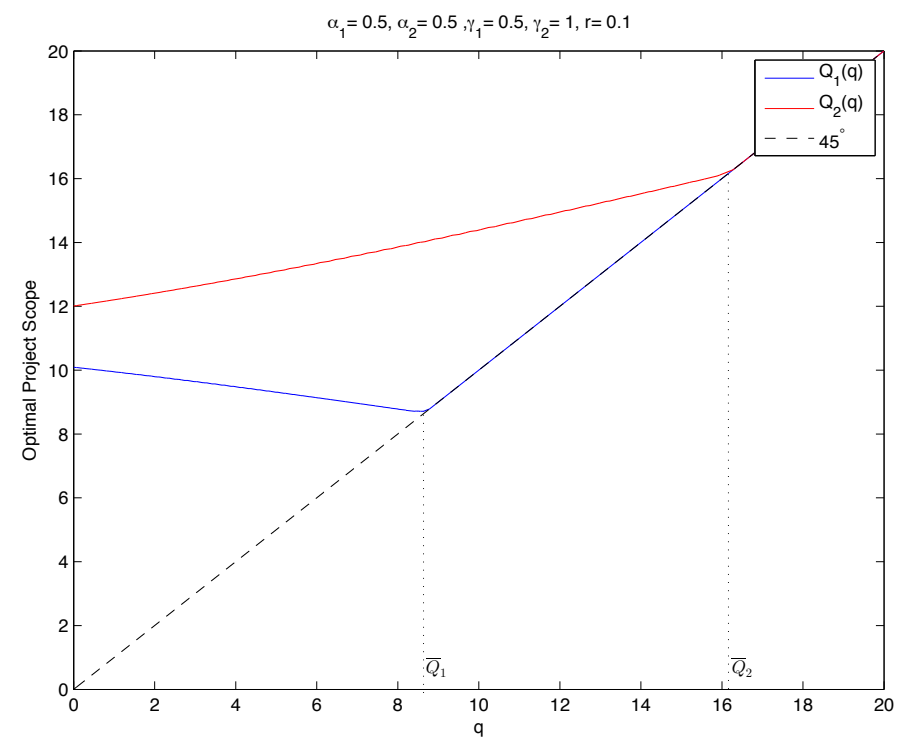

Figure 2: Agent $i$ 's optimal project scope $Q_{i}(q)$

\section{Agents working independently}

In this section, we consider the case in which agent $i$ works alone on the project, and we characterize his optimal project scope. We use this to characterize the equilibrium with endogenous project scope in Section 4. Let $\widehat{J}_{i}(q, Q)$ denote agent $i$ 's discounted payoff function when he works alone on the project, the project scope is $Q$, and he receives $\alpha_{i} Q$ upon completion. ${ }^{20}$ We define agent $i$ 's optimal project scope as

$$
\widehat{Q}_{i}(q)=\arg \max _{Q \geq q}\left\{\widehat{J}_{i}(q ; Q)\right\} .
$$

The following lemma characterizes $\widehat{Q}_{i}(q)$.

Lemma 1. Suppose that agent $i$ works alone on the project. Then his optimal project scope satisfies

$$
\widehat{Q}_{i}(q)=\frac{\alpha_{i}}{2 r \gamma_{i}}
$$

if $q<\frac{\alpha_{i}}{2 r \gamma_{i}}$, and wants to stop the project immediately otherwise. Moreover,

$$
\frac{\alpha_{2}}{2 r \gamma_{2}}<\frac{\alpha_{1}}{2 r \gamma_{1}}<Q_{1}(q)<Q_{2}(q)
$$

\footnotetext{
${ }^{20}$ The value of $\widehat{J}_{i}(q ; Q)$ is given in the proof of Lemma 1 in the Appendix.
} 
for all $q$.

Lemma 1 implies that if an agent works in isolation, then his preferences over the scope are time-consistent when he does not want to stop immediately. Intuitively, when the agent works alone, he bears the entire cost to complete the project, in contrast to the case in which the two agents work jointly. The second part of this lemma rank-orders the agents' ideal project scopes. If an agent works in isolation, then he cannot rely on the other to carry out any part of the project, and therefore the less efficient agent prefers a smaller project scope than the more efficient one. Last, it is intuitive that the more efficient agent's ideal project scope is larger when he works with the other agent relative to when he works alone. As the preferences are time consistent when the agent does not want to stop immediately, we abuse notation and write $\widehat{Q}_{i}=\frac{\alpha_{i}}{2 r \gamma_{i}}$.

\subsection{The social planner}

\section{Social planner's project scope with equilibrium effort level}

We consider a social planner choosing the project scope that maximizes the sum of discounted payoffs, conditional on the agents choosing effort strategically. For this analysis, we assume that the social planner cannot coerce the agents to exert effort, but she can dictate the state at which the project is completed. Thus, the social planner is unable to completely overcome the free-rider problem. Let

$$
Q^{*}(q)=\arg \max _{Q \geq q}\left\{J_{1}(q ; Q)+J_{2}(q ; Q)\right\}
$$

denote the project scope that maximizes the agents' total discounted payoff.

Lemma 2. The project scope that maximizes the agents' total discounted payoff is $Q^{*}(q) \in\left(Q_{1}(q), Q_{2}(q)\right)$.

Lemma 2 states that the social planner's optimal project scope $Q^{*}(q)$ lies between the agents' optimal project scopes for every state of the project. The efficient agent anticipates working harder than the inefficient agent, and hence he wishes to complete the project sooner than is optimal from the planner's perspective. On the other hand, the inefficient agent wishes to complete the project later than optimal. Note that in general, $Q^{*}(q)$ is dependent on $q$; i.e., the social planner's optimal project scope is also time-inconsistent. We illustrate lemmas 1 and 2 in Figure 3 below. 


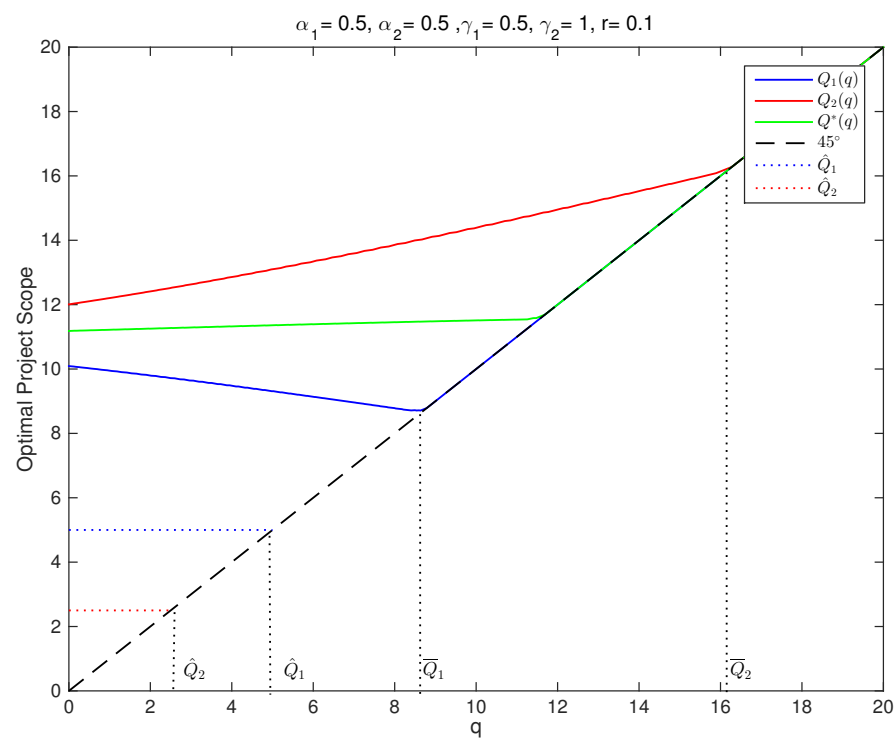

Figure 3: Social planner's project scope $Q^{*}(q)$

\section{Social planner's project scope and effort level}

A classic benchmark of the literature is the cooperative environment in which agents follow the social planner's recommendations for effort. While we focus on the equilibrium project scope more than the free-riding that occurs among agents, we present, for completeness, the solution when the social planner chooses both the agents' level of effort and the project scope.

For a fixed project scope $Q$, the social planner's relevant HJB equation is

$$
r S(q)=\max _{a_{1}, a_{2}}\left\{-\frac{\gamma_{1}}{2} a_{1}^{2}-\frac{\gamma_{2}}{2} a_{2}^{2}+\left(a_{1}+a_{2}\right) S^{\prime}(q)\right\},
$$

subject to $S(Q)=Q$. Each agent's first-order condition is $a_{i}=\frac{S^{\prime}(q)}{\gamma_{i}}$, and substituting this into the HJB equation, we obtain the ordinary differential equation $r S(q)=$ $\frac{\gamma_{1}+\gamma_{2}}{2 \gamma_{1} \gamma_{2}}\left[S^{\prime}(q)\right]^{2}$. This admits the closed form solution for the social planner's value function $S(q)=\frac{r \gamma_{1} \gamma_{2}}{2\left(\gamma_{1}+\gamma_{2}\right)}(q-C)^{2}$, where $C=Q-\sqrt{\frac{2 Q\left(\gamma_{1}+\gamma_{2}\right)\left(\alpha_{1}+\alpha_{2}\right)}{r \gamma_{1} \gamma_{2}}}$. Agent $i$ 's effort level is thus $a_{i}(q)=\frac{r \gamma_{-i}}{\gamma_{1}+\gamma_{2}}(q-C)$. Note that $a_{1}(q)>a_{2}(q)$ for all $q$. That is, the social planner would have the efficient agent do the majority of the work. It is straightforward to show that the social planner's discounted payoff function is maximized at

$$
Q=\frac{\left(\gamma_{1}+\gamma_{2}\right)\left(\alpha_{1}+\alpha_{2}\right)}{2 r \gamma_{1} \gamma_{2}}
$$

at every state of the project, and thus, the planner's preferences are time-consistent. 
This is intuitive, as the time-inconsistency problem is due to the agents not internalizing the externality of their actions and choices. However, as it is unlikely that a social planner can coerce agents to exert a specific amount of effort, we use the result in Lemma 2 as the appropriate benchmark.

\section{Endogenous Project Scope: Real versus Formal Authority}

We now allow agents to choose the project scope by collective choice and discuss the implications for real and formal authority. The project scope in this section is thus endogenous, in contrast to the analysis in Section 3.

As mentioned in the Introduction, our notions of real and formal authority are much like those described in Aghion and Tirole (1997). We consider formal authority to be enforceable by courts, and in this public-project context, an agent has formal authority if he has the right to "sign the documents" or "pull the plug." The collective choice institution thus determines formal authority. We say that agent $i$ has formal authority if he is the dictator. No agent has formal authority if the collective choice institution is unanimity. As pointed out in Aghion and Tirole (1997), the agent endowed with formal authority is not necessarily able to control the project. As an example, consider a developed country assisting a developing country to construct a large infrastructure project. The project, being carried out on the developing country's soil, is subject to its laws and jurisdiction. The developing country thus has formal authority over the project and can specify the termination state, but it is not clear that the developing country does so at a state that is its ideal scope, due to the incentives of the donor developed country. The agent who has control over project scope, and can thus impose his ideal, is said to have real authority. We define real authority precisely as follows.

Definition 1. If the equilibrium project scope $Q$ is decided when the state of the project is $q$, and $Q$ satisfies $Q=Q_{i}(q)$, then agent $i$ has real authority.

In words, we say that an agent has real authority if, at the moment the project scope is decided, it is that agent's most preferred project scope. Recall from Section 3.2 that the agents' preferences over project scope are time-inconsistent. Therefore, today's ideal project scope is no longer ideal tomorrow. Authority thus has a temporal 
component - agent $i$ can only have real authority if the chosen scope is his ideal at the moment it is chosen. Note also that by this definition and Proposition 3.2, if agents are not identical, then at most one agent can have real authority in equilibrium. ${ }^{21} \mathrm{We}$ show that the asymmetry in the agents' effort costs and project stakes, together with the ability to commit, play important roles in determining real authority.

Below we characterize the equilibrium project scope under dictatorship and unanimity, with and without commitment. The equilibria we characterize here are for models that differ from the model with an exogenous project scope in Section 3, and thus uniqueness of MPEs is not assured. Indeed there are cases with multiple MPEs. In such cases, we focus on the equilibrium that maximizes ex-ante total surplus among all MPEs (and naturally is also on the Pareto frontier). Henceforth, when we write equilibrium, we mean ex-ante-surplus-maximizing Markov equilibrium, unless specified otherwise.

\subsection{Dictatorship}

In this section, one of the two agents, denoted agent $i$, has dictatorship rights. He sets the project scope and thus agent $i$ has formal authority. The other agent, agent $j$, can contribute to the project, but has no power to end it. We consider that the dictator can either commit to the project scope or not.

\section{Dictatorship with Commitment}

We first consider dictatorship with commitment. In this institution the dictator can decide at any time to announce a particular project scope, and, following this announcement, the project scope is set once and for all, i.e., neither agent can change it.

If both agents contribute enough, then the project is completed and each agent obtains his reward. If agents do not make sufficient contributions, then the project is never completed: both agents incur the cost of their effort, but neither gets any benefit

\footnotetext{
${ }^{21}$ There may be other ways to think of real authority that can include the possibility that both agents have real authority in equilibrium. For example, if in equilibrium the project is completed at $Q$, where $Q$ is below agent 2's ideal scope and above agent 1's ideal scope, so that neither agent obtains his ideal, we may say that both agents have some degree of real authority. By defining unanimity as both agents having formal authority (rather than neither), the results as summarized in Table 1 are equivalent.
} 
from the project. The project cannot be completed before the dictator announces the project scope.

A strategy for agent $i$ (the dictator) is a pair of maps $\left\{a_{i}(q, Q), \theta_{i}(q)\right\}$ defined for $q \in \mathbb{R}_{+}$and $Q \in \mathbb{R}_{+} \cup\{-1\}$. For $Q \geq 0$, the value $a_{i}(q, Q)$ gives the dictator's effort level in project state $q$ when project scope $Q$ has been decided, and the value $a_{i}(q,-1)$ gives the dictator's effort level in state $q$ if no decision has been made at that state yet. The value $\theta_{i}(q)$ gives the dictator's choice of project scope in state $q$, which applies under the assumption that no project scope has been decided before state $q$ (once a project scope has been set, it is definitive, so the dependence of $\theta_{i}$ on $Q$ is obsolete). We set by convention $\theta_{i}(q)=-1$ if the dictator does not yet wish to commit to a project scope at state $q$, and $\theta_{i}(q) \geq q$ otherwise. Similarly, a strategy for agent $j$ is a map $a_{j}(q, Q)$ associated with his effort level in state $q$ and the project scope decided by the dictator, $Q$ (if $Q \geq 0$ ) or associated with his effort level in state $q$ when no decision has been made yet (if $Q=-1$ ).

The following proposition characterizes the equilibrium. Under commitment, each agent finds it optimal to impose his ideal project scope. The time inconsistency of the dictator's preferences implies that the project scope is always imposed when the project begins, i.e. when $t=0$.

Proposition 4. Under dictatorship with commitment, agent $i$ commits to his ex-ante ideal project scope $Q_{i}(0)$ at the beginning of the game, and the project is completed. Furthermore, this is true in any Markov perfect equilibrium; surplus-maximizing or otherwise. Thus, agent $i$ has real and formal authority.

\section{Dictatorship without Commitment}

We now consider dictatorship without commitment. In this institution, the dictator does not have the ability to credibly commit to a particular project scope. At every instant, he must decide whether to complete the project immediately or continue one more instant. When the decision to complete the project is made, both agents collect the payoffs from project completion. ${ }^{22}$

We define a strategy for agent $i$ (the dictator) as a pair of maps $\left\{a_{i}(q), \theta_{i}(q)\right\}$,

\footnotetext{
${ }^{22}$ Any announcement of project scope other than the current state cannot be committed to. Thus any announcement by agent $i$ other than the current state is ignored by agent $j$ in equilibrium. Since this is the case, agent $i$ 's equilibrium strategy collapses to an announcement to complete the project immediately, or keep working.
} 
where $a_{i}(q)$ determines the effort level of agent $i$ in project state $q, \theta_{i}(q)=-1$ if the agent chooses to continue the project beyond state $q$, and $\theta_{i}(q)=q$ if he chooses to stop the project. A strategy for agent $j$ is a single map $a_{j}(q)$ that determines the agent's effort level in project state $q$.

In the case of dictatorship without commitment, real authority is different from formal authority. Note that $Q^{*}(0)$ is the project scope that maximizes the ex-ante total surplus among all the project scopes. That is, $Q^{*}(0)$ is the social planner's project scope when the state of the project is $q=0$. Recall also that $\bar{Q}_{1}$ is the smallest project scope such that agent 1 , who is the most efficient agent, is indifferent between pursuing the project to a larger scope and terminating it at scope $\bar{Q}_{1}$. We present the equilibrium project scope in Proposition 5 and summarize the implications for real and formal authority in Corollary 1.

Proposition 5. Under dictatorship without commitment, if agent 1 is the dictator, then the equilibrium project scope is $\bar{Q}_{1}$. If agent 2 is the dictator, then the equilibrium project scope is $Q^{*}(0)$.

We provide a heuristic proof, which is useful for understanding the intuition for the result. First, note from Proposition 3 and Lemma 2 that $\bar{Q}_{1}<Q_{1}(0)<Q^{*}(0)<$ $Q_{2}(0)<\bar{Q}_{2}$. When the state is $q=0$, the social planner's project scope is between the agents' ideal project scopes. Recall also from Lemma 1 that $\widehat{Q}_{2}<\widehat{Q}_{1}<\bar{Q}_{1}<\bar{Q}_{2}$.

Conjecture the following strategies when agent 1 is dictator. Agent 1 stops the project immediately when $q \geq \bar{Q}_{1}$ and makes no decision before that. Both agents exert effort according to the MPE with fixed project scope $\bar{Q}_{1}$ when $q \leq \bar{Q}_{1}$ and exert no effort thereafter. We show there is no incentive to deviate from such strategies. Agents' efforts constitute an MPE for a fixed project scope $\bar{Q}_{1}$. Thus, agents have no incentive to exert more or less effort before $\bar{Q}_{1}$. For any $q \leq \bar{Q}_{1}$, agent 1 prefers to continue the project, so there is no incentive to stop the project before that state. Consider $q>\bar{Q}_{1}$. Consider agent 1's incentive to deviate by changing the project scope and exerting strictly positive effort beyond $\bar{Q}_{1}$. In equilibrium, agent 2 exerts no effort beyond $\bar{Q}_{1}$, so anticipating that he will be working alone for all $q>\bar{Q}_{1}$ and noting that $\bar{Q}_{1}>\widehat{Q}_{1}$, agent 1 finds it optimal to complete the project at $\bar{Q}_{1}$.

Next, we consider the case in which agent 2 is dictator and conjecture the following strategies. Agent 2 completes the project at $Q^{*}(0)$ for all $q \leq Q^{*}(0)$, both agents exert the efforts that constitute an MPE for fixed project scope $Q^{*}(0)$, and otherwise they 
exert zero effort for all $q>Q^{*}(0)$. We argue that neither agent has an incentive to deviate, and hence these strategies constitute an equilibrium. As in the previous case, for any $q \geq Q^{*}(0)$, agent 1 has no incentive to exert strictly positive effort because agent 2 completes the project at $q=Q^{*}(0)$. Agent 2 expects to work alone for any $q \geq Q^{*}(0)$, and because $Q^{*}(0)>\widehat{Q}_{2}$, he cannot benefit from delaying the completion of the project and thus has no incentive to deviate. Finally, it follows from Proposition 1 that the agents' effort strategies for $q<Q^{*}(0)$ constitute an MPE.

The prior description of strategies suggests that any project scope in $\left[\widehat{Q}_{i}, \bar{Q}_{i}\right]$ may be an equilibrium project scope when agent $i$ is dictator. Noting that the total surplus of the agents' increases in the project scope for all $Q \leq Q^{*}(0)$, it follows that the unique ex-ante-surplus-maximizing equilibrium project scopes for agents 1 and 2 are $\bar{Q}_{1}$ and $Q^{*}(0)$, respectively.

Under dictatorship without commitment, the asymmetry between the agents becomes important in determining real authority. Recall that agent 2 as dictator can achieve the ex-ante total surplus-maximizing project scope $Q^{*}(0)$ in equilibrium, but agent 1 as dictator cannot. In particular, agent 1 desires a smaller project scope than agent 2 at every state, and as dictator, he can complete the project regardless of agent 2's desire to continue. Therefore, as dictator, agent 1 has both real and formal authority. On the other hand, agent 2 desires a larger project than agent 1 at every state, so his decision to complete the project depends on his expectations about agent 1 exerting strictly positive effort. As a result, upon completion of the project at $Q^{*}(0)$, agent 1 desires to stop, but agent 2 would like to continue (provided that agent 1 exerted effort). Therefore, even if agent 2 has formal authority, it is agent 1 who has real authority.

Corollary 1 (Formal, but not real authority). Under no commitment, if agent 1 is the dictator, then he has real and formal authority. If agent 2 is the dictator, then he has formal authority but not real authority, and instead agent 1 has real authority.

\subsection{Unanimity}

In this section, we consider the case in which both agents must agree on the project scope. We say that neither agent has formal authority in this case. One of the agents, whom we denote by $i$, is (exogenously) chosen to be the agenda setter. He makes a proposal for the project scope. The other agent (agent $j$ ) must respond to the agenda 
setter's proposal by either accepting or rejecting the proposal. ${ }^{23}$ If the proposal is rejected, then no decision is made about the project scope. The project will not be completed until a decision is made about the project scope.

As in the dictatorship case analyzed in the previous section, we will consider both the case in which the agenda setter can commit to the proposed project scope and the case where he cannot commit.

\section{Unanimity with Commitment}

We first consider unanimity with commitment. In this case, at any instant, the agenda setter can propose a project scope. Upon proposal, the other agent must decide to either accept or reject the offer. If he accepts, then the project scope agreed upon is set once and for all and cannot be changed. From that instant onwards, the agenda setter stops making proposals. The agents may continue to work on the project, and the project is completed if, and only if, the project state reaches the agreed upon project scope. At this time, the agents get payoffs from project completion. If agent $j$ rejects the proposal, then no project scope is decided upon, and the agenda setter may continue to make further proposals. The project cannot be completed until a project scope proposed by agent $i$ is agreed upon by agent $j$.

A strategy for the agenda setter is a pair $\left\{a_{i}(q, Q), \theta_{i}(q)\right\}$. Here, $a_{i}(q, Q)$ denotes the effort level of the agenda setter when the project state is $q$ and the project scope agreed upon is $Q$; by convention, $Q=-1$ if no agreement has been reached yet. The value of $\theta_{i}(q)$ is the project scope proposed by the agenda setter in project state $q$; by convention, $\theta_{i}(q)=-1$ if the agent does not wish to make a proposal at that time. A Markov strategy for agent $j$ is a pair of maps $\left\{a_{j}(q, Q), Y_{j}(q, Q)\right\}$. Similarly, the map $a_{j}(q, Q)$ denotes the effort level in state $q$ when project scope $Q$ has already been agreed upon, and as above, $a_{j}(q,-1)$ is agent $j$ 's effort level when no agreement has been reached yet. The map $Y_{j}(q, Q)$ is the acceptance strategy of agent $j$ if agent $i$ proposes project scope $Q$ at state $q$, where $Y_{j}(q, Q)=1$ if agent $j$ accepts, while $Y_{j}(q, Q)=0$ if he rejects.

Proposition 6. Under unanimity with commitment, the equilibrium project scope is $Q^{*}(0)$. The project scope is decided at the beginning of the project, and neither agent has real authority.

\footnotetext{
${ }^{23}$ The equilibrium project scope is independent of who is the agenda-setter.
} 


\section{Unanimity without Commitment}

We now study the case in which the agenda setter cannot commit. The agenda setter can make a proposal to complete the project at any time he wishes. Upon proposal, agent $j$ must decide to accept or reject. If he accepts, the project is completed immediately, and both agents obtain their payoffs. If agent $j$ rejects the proposal, both agents may continue to work on the project, and agent $i$ can make further proposals. The project cannot be completed and agents do not get payoffs from completion until agent $j$ agrees to an offer from the agenda setter. ${ }^{24}$

A Markov strategy for the agenda setter is a pair $\left\{a_{i}(q), \theta_{i}(q)\right\}$, where as before, $a_{i}(q)$ is the effort level of the agent in project state $q$, while $\theta_{i}(q)$ indicates whether the agent makes a proposal to complete the project: $\theta_{i}(q)=q$ if he makes such a proposal, and $\theta_{i}(q)=-1$ otherwise. A Markov strategy for agent $j$ is a pair $\left\{a_{j}(q), Y_{j}(q)\right\}$, where $a_{j}(q)$ records the effort level in state $q$, while $Y_{j}(q)$ records the response of agent $j$ in the event of a proposal made by the agenda setter in state $q: Y_{j}(q)=1$ if agent $j$ agrees to stop the project in state $q$, and $Y_{j}(q)=0$ otherwise. ${ }^{25}$ Note that, as opposed to the commitment case, the strategies no longer condition on any agreed upon project scope $Q$, as no agreement on the project scope is reached before the project is completed.

Proposition 7. Under unanimity without commitment, the equilibrium project scope is $Q^{*}(0)$. When the project is completed at $Q^{*}(0)$, it is agent 1's ideal, and thus agent 1 has real authority.

The equilibria of these games shed light on who has real authority to decide the scope of a public project. Under commitment, real and formal authority are equivalent. Under no commitment, if agent 1 is dictator, then he has both real and formal

\footnotetext{
${ }^{24}$ In contrast to the commitment case, the agenda setter cannot propose a project scope to be agreed upon. This is to simplify the exposition; however, the results would continue to hold if the agenda setter were to make (non-binding) project-scope proposals. Without the ability to commit to completing the project at some future state, proposing any scope greater that the current state is only equivalent to continuing the project towards some undecided project scope, with or without agreement from the other agent. The extra communication does not impact equilibrium outcomes given our focus on MPEs.

${ }^{25}$ Alternatively, agent $j$ may be required to agree to continue the project. It can be shown that the unique (surplus-maximizing) equilibrium project scope is $\bar{Q}_{1}$ with this assumption, i.e., the same equilibrium project scope reached when agent 1 is dictator without commitment. A proof is available upon request. Note that, with this assumption, agent 1 still has real authority under unanimity. We assume agents must agree to terminate the project in the no-commitment case to be consistent with the commitment case.
} 
authority. On the other hand, if agent 2 is dictator, then he has formal authority but not real authority. With no commitment, real authority is thus not equivalent to formal authority. Table 1 below summarizes these results, where D1 (D2) indicates that agent 1 (agent 2) is dictator, and $U$ refers to unanimity with either agent as agenda setter.

\begin{tabular}{|c||c|c|c|}
\cline { 2 - 4 } \multicolumn{1}{c|}{} & \multicolumn{3}{c|}{ Institution } \\
\cline { 2 - 4 } \multicolumn{1}{c|}{} & D1 & D2 & U \\
\hline \hline commitment & agent 1 & agent 2 & neither \\
\hline no commitment & agent 1 & agent 1 & agent 1 \\
\hline
\end{tabular}

Table 1: Agent with real authority

A natural question is which collective choice institution admits the social planner's project scope as an equilibrium outcome. First, note that when the efficient agent is dictator, the planner's project scope cannot be part of an equilibrium regardless of the ability to commit ex-ante. On the other hand, if the inefficient agent is dictator and he cannot commit ex-ante, then the planner's project scope can be implemented in equilibrium. Finally, under unanimity, the planner's project scope is an equilibrium outcome both with and without commitment. We summarize these results in Table 2 and formally in Corollary 2.

\begin{tabular}{|c|c|c|c|}
\cline { 2 - 4 } \multicolumn{1}{c|}{} & \multicolumn{3}{c|}{ Institution } \\
\cline { 2 - 4 } \multicolumn{1}{c|}{} & D1 & D2 & U \\
\hline \hline commitment & too low & too high & equal \\
\hline no commitment & too low & equal & equal \\
\hline
\end{tabular}

Table 2: Equilibrium project scope relative to social planner's ideal project scope

Corollary 2 (Optimality). With commitment, the social planner's ex-ante ideal project scope can only be implemented with unanimity. Without commitment, the social planner's project scope can be implemented when the inefficient agent is dictator or with unanimity.

Note that only unanimity can deliver the social planner's project scope both with and without commitment. In this sense, unanimity dominates dictatorship. The 
dominance of unanimity with no commitment, while allowing the efficient agent to retain real authority, may help explain why it is often the case that agreements formally governed by unanimity still appear to be heavily influenced by large contributors. These large donors are the more efficient agents, who contribute more to the public project and hence have the incentive to stop the project before the inefficient agent.

\section{Extensions}

In this section, we consider two extensions of our main model. We first allow agents to use transfers, and then consider the case in which the project progresses stochastically.

\subsection{Transfers}

So far we have assumed that each agent's project stake $\alpha_{i}$ is exogenous, and transfers are not permitted. These are reasonable assumptions if agents are liquidity constrained. However, if transfers are available, there are various ways to mitigate the inefficiencies associated with the collective choice problem. Our objective in this section is to shed light on how transfers can be useful for improving the efficiency properties of the collective choice institutions. We consider that agents choose effort levels strategically, so free-riding still occurs. We look at two types of transfers. First, we discuss the possibility that the agents can make lump-sum transfers at the beginning of the game to directly influence the project scope that is implemented. Second, we consider the case in which the agents can bargain over the allocation of shares in the project in exchange for transfers. In both cases, we assume that the agents commit to the project scope, transfers, and reallocation of shares at the outset of the game.

\section{Transfers contingent on project scope}

We first consider the case in which one of the agents is dictator, and he can commit to a particular project scope. Assume that agent 1 is dictator and makes a take-it-orleave-it offer to agent 2, which specifies a transfer in exchange for committing to some project scope $Q$. Recall that $J_{k}(q ; Q)$ denotes agent $k$ 's value function at project state 
$q$ when the project scope is $Q$. Then agent 1 solves the following problem:

$$
\begin{aligned}
\max _{Q \geq 0, T \in \mathbb{R}} & J_{1}(0 ; Q)-T \\
\text { s.t. } & J_{2}(0 ; Q)+T \geq J_{2}\left(0 ; Q_{1}(0)\right) .
\end{aligned}
$$

In words, agent 1 chooses the project scope and the corresponding transfer to maximize his ex-ante discounted payoff, subject to agent 2 obtaining a payoff that is at least as great as his payoff if he were to reject agent 1's offer, in which case agent 1 would commit to the status quo project scope $Q_{1}(0)$, and no transfer would be made. Because transfers are unlimited, the constraint binds in the optimal solution, and the problem reduces to

$$
\max _{Q \geq 0}\left\{J_{1}(0 ; Q)+J_{2}(0 ; Q)-J_{2}\left(0 ; Q_{1}(0)\right)\right\} .
$$

Note that the optimal choice of $Q$ maximizes total surplus. This is intuitive: because the agents have complete and symmetric information, bargaining is efficient. It is straightforward to verify that the same result holds under any one-shot bargaining protocol irrespective of which agent has dictatorship rights, and for any initial status quo. $^{26}$

\section{Transfers contingent on reallocation of shares}

We now consider $\alpha_{1}+\alpha_{2}=1$, so the project stakes can be interpreted as project shares. We consider an extension of the model in which, at the outset, the agents start with an exogenous allocation of shares and then engage in a bargaining game in which shares can be reallocated in exchange for a transfer. After the allocation of shares, the collective choice institution determines the choice of scope as given in Section 4 . Note that the allocation of shares influences the agents' incentives and consequently the equilibrium project scope. Because this is a game with complete information, the agents reallocate the shares so as to maximize the ex-ante total discounted surplus, taking the collective choice institution as given.

Based on the analysis of Section 4, there are the following cases to consider:

1. Agent $i$ is dictator, for $i \in\{1,2\}$, and he has the ability to commit. As such, he

\footnotetext{
${ }^{26}$ One might also consider the case in which commitment is not possible. Because $Q_{1}(q) \leq Q_{2}(q)$ for all $q$, to influence the project scope at some state, agent 1 might offer a lump-sum transfer to agent 2 in exchange for completing the project immediately, whereas agent 2 might offer flow transfers to agent 1 to extend the scope of the project. This model is intractable, so we do not pursue it in the current paper.
} 
commits to $Q=Q_{i}(0)$ at the outset, by Proposition 4 .

2. Agent 1 is dictator, but he is unable to commit. In this case, the project is completed at state $\bar{Q}_{1}$, by Proposition 5 .

3. Agent 2 is dictator, but he is unable to commit, or decisions must be made unanimously, with or without commitment. In these cases, the equilibrium project scope is $Q^{*}(0)$ by Propositions 5,6 , and 7 , respectively.

We focus the analysis on the case in which agent 1 is dictator and can commit to a particular project scope at the outset; the other cases lead to similar insights. To begin, let $Q_{1}(0 ; \alpha)$ denote the (unique) equilibrium project scope when agent 1 is dictator and has the ability to commit, conditional on the shares $\left\{\alpha_{1}, 1-\alpha_{1}\right\}$. Assume that agent 1 makes a take-it-or-leave-it offer to agent 2 , which specifies a transfer in exchange for reallocating the parties' shares from the status quo shares $\left\{\bar{\alpha}_{1}, 1-\bar{\alpha}_{1}\right\}$ to $\left\{\alpha_{1}, 1-\alpha_{1}\right\}$. Let $J_{k}(q ; Q, \alpha)$ be the continuation value for agent $k$ when the state is $q$, the chosen project scope is $Q$ and the chosen share to agent 1 is $\alpha$. Then agent 1 solves the following problem:

$$
\begin{aligned}
\max _{\alpha_{1} \in[0,1], T \in \mathbb{R}} & J_{1}\left(0 ; Q_{1}\left(0 ; \alpha_{1}\right), \alpha_{1}\right)-T \\
\text { s.t. } & J_{2}\left(0 ; Q_{1}\left(0 ; \alpha_{1}\right), \alpha_{1}\right)+T \geq J_{2}\left(0 ; Q_{1}\left(0 ; \bar{\alpha}_{1}\right), \bar{\alpha}_{1}\right) .
\end{aligned}
$$

Because transfers are unlimited and each agent's discounted payoff increases in his share, the incentive compatibility constraint binds in the optimal solution, and so the problem reduces to

$$
\max _{\alpha_{1} \in[0,1]}\left\{J_{1}\left(0 ; Q_{1}\left(0 ; \alpha_{1}\right), \alpha_{1}\right)+J_{2}\left(0 ; Q_{1}\left(0 ; \alpha_{1}\right), \alpha_{1}\right)-J_{2}\left(0 ; Q_{1}\left(0 ; \bar{\alpha}_{1}\right), \bar{\alpha}_{1}\right)\right\} .
$$

The optimal choice of $\alpha_{1}$ maximizes total surplus, conditional on the scope subsequently selected by the collective choice institution. In all other cases, and under any one-shot bargaining protocol, the agents will agree to re-allocate their shares to maximize total surplus.

The problem of optimally reallocating shares is analytically intractable, therefore we find the solution numerically. Figure 4 below illustrates the share allocated to agent 1, as a function of his effort cost, and Figure 5 gives the total surplus. Note that without commitment, both the case of unanimity and the case in which agent 2 is dictator deliver the same result, and hence the result for unanimity are omitted. 


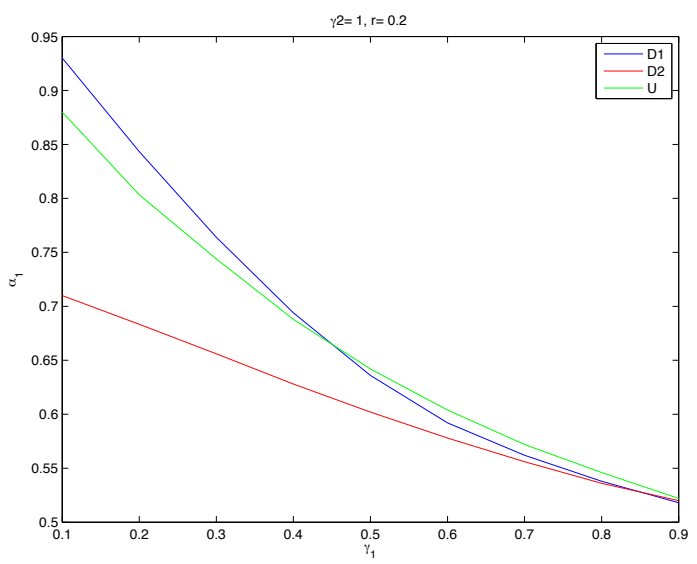

(a) Commitment

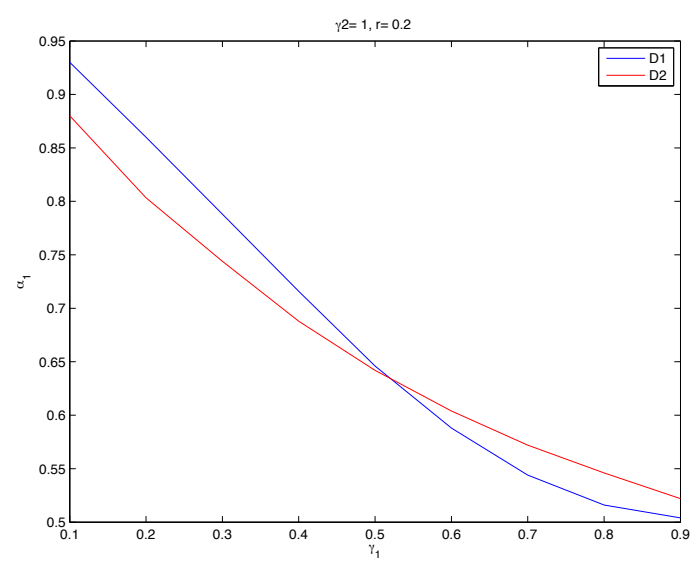

(b) No commitment

Figure 4: Agent 1's optimal project share

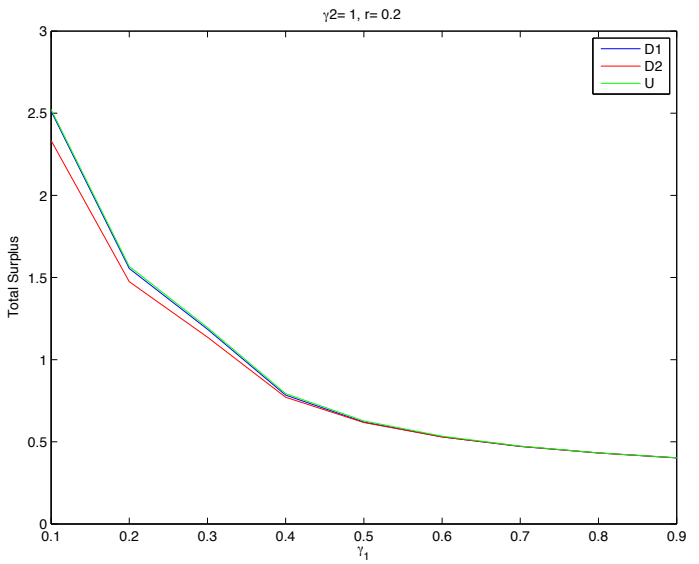

(a) Commitment

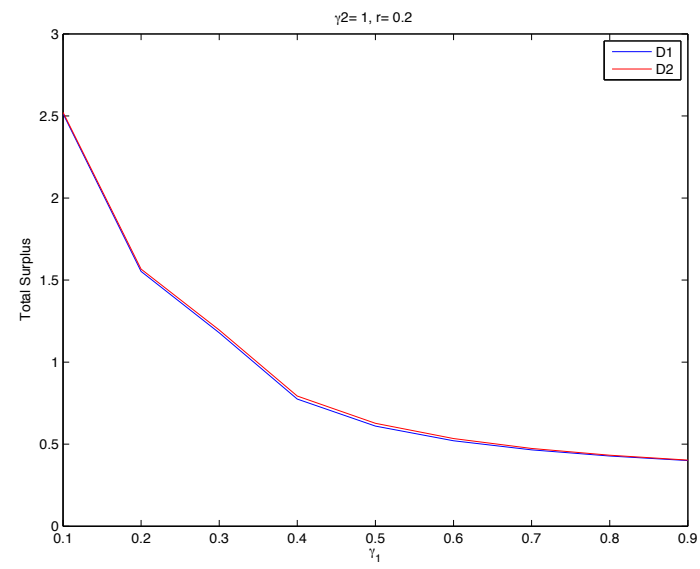

(b) No commitment

Figure 5: Total surplus

In all cases, it is optimal for agent 1 , who is more productive, to possess the majority of the shares. Moreover, his optimal allocation decreases as his effort costs increase, i.e., as he becomes less productive. In other words, if one agent is substantially more productive than the other, then the former should possess the vast majority of the shares. Indeed, it is efficient to provide the stronger incentives to the more productive agent, and the smaller the disparity in productivity between the agents, the smaller should be the difference in the shares that they possess.

With commitment, total surplus is highest under unanimity. Absent the ability to commit, unanimity or agent 2 as dictator achieves the social planner's surplus, and agent 1 as dictator cannot do better. That is because the (ex-ante) surplus-maximizing 
project scope is in the set of MPEs under those institutions, and by our refinement, it is the one that is implemented. One exception is when the agents have identical effort costs, in which case all collective choice institutions lead to the same total surplus, and it is optimal for the two agents to split the shares equally (i.e., $\alpha_{1}=\alpha_{2}=\frac{1}{2}$ ).

With commitment and dictatorship, total surplus is greater if agent 1 is dictator (compared with agent 2 being dictator) if his effort costs are sufficiently small relative to agent 2's. Intuitively, the agent who has formal authority gets to implement his ideal project scope, so he has stronger incentives to exert effort. Therefore, if agent 1 is significantly more productive than agent 2 , then total surplus is higher if he is conferred formal authority. The opposite is true if the agents differ only marginally in their productivity.

\subsection{Collective Choice under Uncertainty}

To obtain tractable results, we have assumed that the project progresses deterministically. To examine the robustness of our results to this assumption, we consider the case in which the project progresses stochastically according to

$$
d q_{t}=\left(a_{1 t}+a_{2 t}\right) d t+\sigma d Z_{t},
$$

where $\sigma>0$ captures the degree of uncertainty associated with the evolution of the project, and $Z_{t}$ is a standard Brownian motion. We discuss the results for collective choice under this form of uncertainty.

As in the deterministic case in Section 3, we begin by establishing the existence of an MPE with an exogenous project scope $Q$. In an MPE, each agent's discounted payoff function satisfies

$$
r J_{i}(q)=\frac{\left[J_{i}^{\prime}(q)\right]^{2}}{2 \gamma_{i}}+\frac{1}{\gamma_{j}} J_{i}^{\prime}(q) J_{j}^{\prime}(q)+\frac{\sigma^{2}}{2} J_{i}^{\prime \prime}(q)
$$

subject to the boundary conditions $\lim _{q \rightarrow-\infty} J_{i}(q)=0$ and $J_{i}(Q)=\alpha_{i} Q$ for each $i$. Using the normalization $\widetilde{J}_{i}(q)=\frac{J_{i}(q)}{\gamma_{i}}$, it is straightforward to show that

$$
r \widetilde{J}_{i}(q)=\frac{\left[\widetilde{J}_{i}^{\prime}(q)\right]^{2}}{2}+\widetilde{J}_{i}^{\prime}(q) \widetilde{J}_{j}^{\prime}(q)+\frac{\sigma^{2}}{2} \widetilde{J}_{i}^{\prime \prime}(q)
$$

subject to $\lim _{q \rightarrow-\infty} \widetilde{J}_{i}(q)=0$ and $\widetilde{J}_{i}(Q)=\frac{\alpha_{i}}{\gamma_{i}} Q$ for each $i$. Agents in this problem have identical per unit effort costs but are asymmetric with respect to their stake 
in the project. It follows from Georgiadis (2015) that an MPE exists and satisfies $\widetilde{J}_{i}(q)>0, \widetilde{J}_{i}^{\prime}(q)>0, a_{i}(q)>0$ and $a_{i}^{\prime}(q)>0$ for all $i$ and $q$. Equivalently, $J_{i}(q)>0$, $J_{i}^{\prime}(q)>0$, and effort choices in the original problem are the same as in the normalized problem. This is the analog of Proposition 1 in the case of uncertainty.

As in the case with no uncertainty, we next establish the key properties of the MPE with exogenous project scope for asymmetric agents.

Proposition 8 (Uncertainty). Consider the model with uncertainty, and suppose that $\frac{\gamma_{1}}{\alpha_{1}}<\frac{\gamma_{2}}{\alpha_{2}}$. In any project-completing MPE:

1. Agent 1 exerts higher effort than agent 2 in every state, and agent 1 's effort increases at a greater rate than agent 2's. That is, $a_{1}(q) \geq a_{2}(q)$ and $a_{i}^{\prime}(q) \geq$ $a_{2}^{\prime}(q)$ for all $q \geq 0$.

2. Agent 1 obtains a lower discounted payoff normalized by project stake than agent 2. That is, $\frac{J_{1}(q)}{\alpha_{1}} \leq \frac{J_{2}(q)}{\alpha_{2}}$ for all $q \geq 0$.

Suppose instead that $\frac{\gamma_{1}}{\alpha_{1}}=\frac{\gamma_{2}}{\alpha_{2}}$. In any project-completing MPE, $a_{1}(q)=a_{2}(q)$ and $\frac{J_{1}(q)}{\alpha_{1}}=\frac{J_{2}(q)}{\alpha_{2}}$ for all $q \geq 0$.

Proposition 8 is the analog of Proposition 2 in the case of uncertainty. It states that, under uncertainty, if agents are asymmetric, the efficient agent exerts higher effort at every state of the project, and the efficient agent's effort increases at a higher rate than that of the inefficient agent. Furthermore, the efficient agent achieves a lower discounted payoff (normalized by the stake $\alpha_{i}$ ) at every state of the project.

As for the case of transfers, this extension is not as tractable as our main model, but numerical computations suggest that the results of Proposition 3.2 continue to hold. This is not surprising given the result in Proposition 8 and because the intuition for the ordering and divergence of preferences is identical to that for the case without uncertainty. An example is illustrated in Figure 6.

As Figure 6 illustrates, the inefficient agent prefers a larger scope than the efficient agent at every state, and furthermore, his ideal project scope increases over the course of the project, whereas the efficient agent's ideal project scope decreases. The social planner's project scope lies between the agents' ideal project scope at every state.

Notice that the results of Section 4 rely on the key properties of the preferences illustrated in Figure 6. Conditional on these preferences, all results of Propositions 4-7 will hold. The proofs follow directly from the proofs of Propositions 4-7, so they are omitted. 


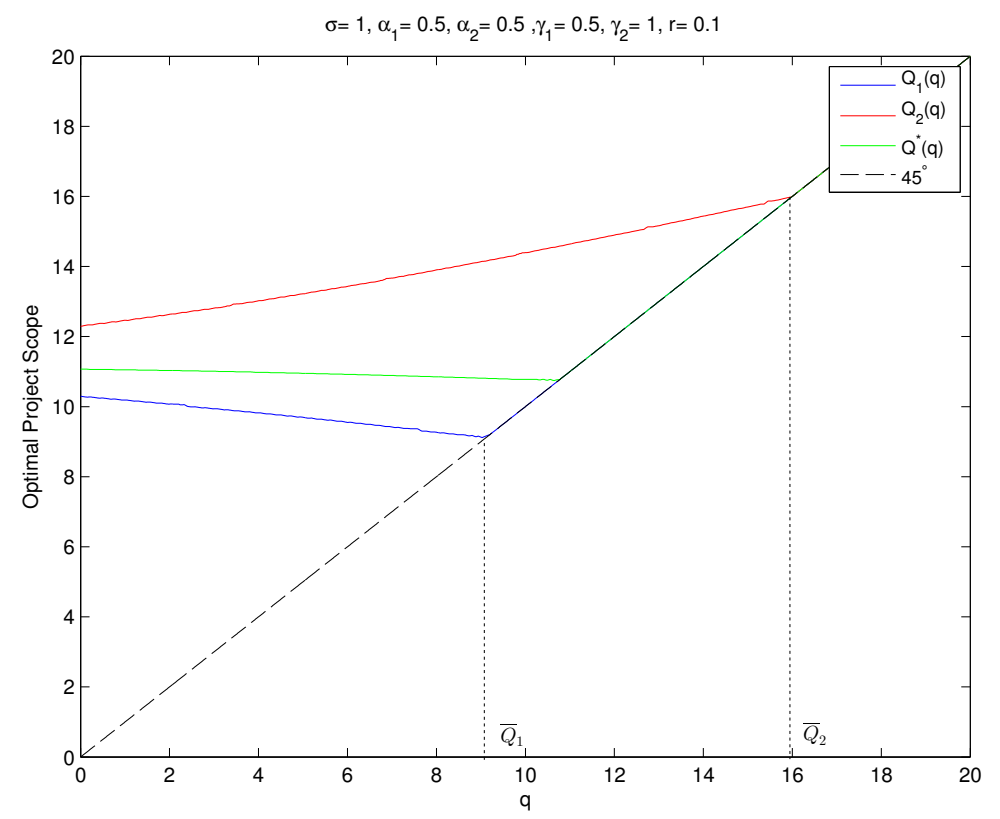

Figure 6: Agent $i$ 's optimal project scope $Q_{i}(q)$ with uncertainty

\section{Conclusion}

In this paper we begin to investigate the determinants of real authority over the scope of a public project and present an efficiency theory of real authority. We study heterogenous agents making costly contributions towards the completion of a public project, when the decision about the project scope can be made at any time. Previous works have studied voluntary contribution games with symmetric agents and documented the dynamic free-rider problem. We show here that asymmetries have important effects in this class of problems. These effects depend on the level of commitment. With commitment, real and formal authority are equivalent, because agents are able to make decisions at the beginning of the project and not change them. Without commitment, however, the agents can only credibly announce to stop the project immediately or keep going, due to the time-inconsistency of preferences. This inability to commit allows the efficient agent to "hold up" the project. Under the threat of no continued effort from the efficient agent, the inefficient agent, even with formal authority or unanimity, discontinues the project at the moment that is best for the efficient agent.

With respect to total surplus, we show that unanimity dominates dictatorship, both with and without commitment. Under unanimity, the social planner's project 
scope is always selected, yet, it allows the efficient agent to retain control when there is no commitment. Interestingly, if formal authority were to be delegated to maximize surplus with no commitment, the inefficient agent as dictator would perform just as well as unanimity and would achieve the social planner's solution. This suggests that some formal authority should be allocated to the inefficient agent, either with unanimity or with the inefficient agent as dictator. Transfers naturally improve welfare under any collective choice institution.

The results suggest several directions for future research. As mentioned in the Introduction, we analyze a two-agent model, whereas many applications of interest have more than two agents. A natural next step is to extend some of these results to a model with an arbitrary number of players and understand, first, how incentives for effort interact, and second, the implications for real and formal authority. With even three players, other collective choice institutions can be considered, such as majority voting. The model assumes the project benefit is deterministic and complete information to the players. This makes the model tractable but likely misses important effects related to learning about the project benefit over time. Further work incorporating uncertainty of this kind would be quite fruitful. We take the simple perspective that an agent's total project benefit is the product of the agent's stake and the project scope. The agent's stake is thus fixed throughout the project. This does not take into account changes in an agent's stake as time progresses, or the fact that the project benefit may be some other function of the stake and project scope. Last, if an agent's cost of effort is private information, then our results suggest that the efficient agent may have an incentive to mimic the inefficient agent, thus contributing a smaller amount of effort. This may lead to a greater ideal project scope for the efficient agent, which will be welfare enhancing if the efficient agent is the dictator, but the welfare implications are not immediate because the distribution of work will likely be further away from that of the social planner. We leave these considerations for future work. 


\section{A Appendix}

\section{A.1 Some Auxiliary Results}

We present below several lemmas that will be used throughout the proofs of the main results. Throughout we consider the benchmark game of Section 3 with exogenous project scope $Q$.

Lemma 3. Let $\left(J_{1}, J_{2}\right)$ be a pair of well-behaved value functions associated with an MPE. Then $J_{i}(q) \in\left[0, \alpha_{i} Q\right]$ and $J_{i}^{\prime}(q) \geq 0$ for all $i$ and $q$.

Proof. Because each agent $i$ can guarantee himself a payoff of zero by not exerting any effort, in any equilibrium, it must be the case that $J_{i}(q) \geq 0$ for all $q$. Moreover, because he receives reward $\alpha_{i} Q$ upon completion of the project, he discounts time, and the cost of effort is nonnegative, his payoff satisfies $J_{i}(q) \leq \alpha_{i} Q$ for all $q$. Next, suppose that $J_{i}^{\prime}\left(q^{*}\right)<0$ for some $i$ and $q^{*}$. Then agent $i$ exerts zero effort at $q^{*}$, and it must be the case that agent $j \neq i$ also exerts zero effort, because otherwise $J_{i}\left(q^{*}\right)<0$, which cannot occur in equilibrium. Since both agents exert zero effort at $q^{*}$, the project is never completed, and so $J_{1}\left(q^{*}\right)=J_{2}\left(q^{*}\right)=0$. Therefore, for sufficiently small $\epsilon>0$, we have $J_{i}\left(q^{*}+\epsilon\right)<0$, which is a contradiction, implying $J_{i}^{\prime}(q) \geq 0$ for all $i$ and $q$.

The following lemma derives an explicit system of ODEs that is equivalent to the implicit form given in (5) of Section 3.

Observe that dividing both sides of equation (3) by $\gamma_{i}$ the system of ODEs defined by (3) subject to (2) can be rewritten as

$$
r \widetilde{J}_{i}(q)=\frac{1}{2}\left[\widetilde{J}_{i}^{\prime}(q)\right]^{2}+\widetilde{J}_{i}^{\prime}(q) \widetilde{J}_{j}^{\prime}(q)
$$

subject to $\widetilde{J}_{i}(Q)=\frac{\alpha_{i}}{\gamma_{i}} Q$ for all $i \in\{1,2\}$ and $j \neq i$.

Lemma 4. Let $\left(J_{1}, J_{2}\right)$ be a pair of well-behaved value functions associated with an

$M P E$, and let $\widetilde{J}_{i}(q)=\frac{J_{i}(q)}{\gamma_{i}}$. Then if, at state $q$, the project is completing, the following 
explicit ODEs are satisfied on the range $(q, Q) \cdot{ }^{27}$

$$
\begin{aligned}
& \widetilde{J}_{1}^{\prime}=\sqrt{\frac{r}{6}} \sqrt{2 \sqrt{\left(\widetilde{J}_{1}\right)^{2}+\left(\widetilde{J}_{2}\right)^{2}-\widetilde{J}_{1} \widetilde{J}_{2}}+\widetilde{J}_{1}+\widetilde{J}_{2}}+\sqrt{\frac{r}{2}} \sqrt{2 \sqrt{\left(\widetilde{J}_{1}\right)^{2}+\left(\widetilde{J}_{2}\right)^{2}-\widetilde{J}_{1} \widetilde{J}_{2}}}-\widetilde{J}_{1}+\widetilde{J}_{2}, \\
& \widetilde{J}_{2}^{\prime}=\sqrt{\frac{r}{6}} \sqrt{2 \sqrt{\left(\widetilde{J}_{1}\right)^{2}+\left(\widetilde{J}_{2}\right)^{2}-\widetilde{J}_{1} \widetilde{J}_{2}}+\widetilde{J}_{1}+\widetilde{J}_{2}}-\sqrt{\frac{r}{2} \sqrt{2 \sqrt{\left(\widetilde{J}_{1}\right)^{2}+\left(\widetilde{J}_{2}\right)^{2}-\widetilde{J}_{1} \widetilde{J}_{2}}}-\widetilde{J}_{1}+\widetilde{J}_{2}} .
\end{aligned}
$$

Proof. In an MPE in which the project is completing at state $q, \widetilde{J}_{1}^{\prime}+\widetilde{J}_{2}^{\prime}>0$ on $[q, Q)$ as otherwise both agents put zero effort at some intermediary state and the project is not completed.

Using (5), subtracting $\widetilde{J}_{2}$ from $\widetilde{J}_{1}$ and adding $\widetilde{J}_{2}$ to $\widetilde{J}_{1}$ yields

$$
\begin{aligned}
r\left(\widetilde{J}_{1}-\widetilde{J}_{2}\right)-\frac{1}{2}\left(\widetilde{J}_{1}^{\prime}+\widetilde{J}_{2}^{\prime}\right)\left(\widetilde{J}_{1}^{\prime}-\widetilde{J}_{2}^{\prime}\right) & =0, \text { and } \\
r\left(\widetilde{J}_{1}+\widetilde{J}_{2}\right)-\frac{1}{2}\left(\widetilde{J}_{1}^{\prime}+\widetilde{J}_{2}^{\prime}\right)^{2} & =\widetilde{J}_{1}^{\prime} \widetilde{J}_{2}^{\prime},
\end{aligned}
$$

respectively, where for notational simplicity we drop the argument $q$. Letting $G=$ $\widetilde{J}_{1}+\widetilde{J}_{2}$ and $F=\widetilde{J}_{1}-\widetilde{J}_{2}$, these equations can be rewritten as

$$
\begin{aligned}
r F-\frac{1}{2} F^{\prime} G^{\prime} & =0 \\
r G-\frac{1}{2}\left(G^{\prime}\right)^{2} & =\frac{1}{4}\left(G^{\prime}\right)^{2}-\frac{1}{4}\left(F^{\prime}\right)^{2} .
\end{aligned}
$$

From the first equation we have $F^{\prime}=\frac{2 r F}{G^{\prime}}$ (and recall that we have assumed $G^{\prime}>0$ ), while the second equation, after plugging in the value of $F^{\prime}$, becomes

$$
r G-\frac{1}{2}\left(G^{\prime}\right)^{2}=\frac{1}{4}\left(G^{\prime}\right)^{2}-r^{2} \frac{F^{2}}{\left(G^{\prime}\right)^{2}},
$$

This equation is quadratic in $\left(G^{\prime}\right)^{2}$, and noting by Lemma 3 that in any projectcompleting MPE we have $G^{\prime}>0$ on $[0, Q]$, the unique strictly positive root is

$$
\left(G^{\prime}\right)^{2}=\frac{2 r}{3}\left(\sqrt{G^{2}+3 F^{2}}+G\right) \Longrightarrow G^{\prime}=\sqrt{\frac{2 r}{3}} \sqrt{\sqrt{G^{2}+3 F^{2}}+G}
$$

Since $G^{\prime}>0$ on the interval of interest, we have

$$
F^{\prime}=\frac{2 r F}{G^{\prime}}=\frac{\sqrt{6 r} F}{\sqrt{\sqrt{G^{2}+3 F^{2}}+G}} \Longrightarrow F^{\prime}=\sqrt{2 r} \sqrt{\sqrt{G^{2}+3 F^{2}}-G} .
$$

By using that $\widetilde{J}_{1}=\frac{1}{2}(G+F)$ and $\widetilde{J}_{2}=\frac{1}{2}(G-F)$, we obtain the desired expressions.

\footnotetext{
${ }^{27}$ We say that the project is completing at state $q$ to indicate that if the state is $q$, then the project will be completed. In contrast, we say that the project is completed at state $Q$ to indicate that state $Q$ is the termination state.
} 
The following result is a direct consequence of Lemma 4.

Lemma 5. Let $\left(J_{1}, J_{2}\right)$ be a pair of well-behaved value functions associated with an $M P E$. Then for every state $q, J_{1}(q)>0$ if and only if $J_{2}(q)>0$. Furthermore, if the project is completing at state $q$, then both $J_{1}^{\prime}$ and $J_{2}^{\prime}$ are strictly positive on $(q, Q)$.

Proof. Fix agent $i$ and let $j$ denote the other agent. If $J_{i}(q)>0$, then the project is completing at state $q$. By Lemma $4, \widetilde{J}_{1}^{\prime}$ is bounded strictly above 0 on $(q, Q)$, thus $J_{1}^{\prime}$ is also bounded strictly above zero on that range, and as an agent's action is proportional to the slope of the value function, agent 1's effort is also bounded strictly above 0 on the range $(q, Q)$. This implies that, if agent 2 chooses to exert no effort on $(q, Q)$, potentially deviating from his equilibrium strategy, the project is still completed by agent 1 -and thus agent 2 makes a strictly positive discounted payoff at state $q$ without exerting any effort from state $q$ onwards. Agent 2's equilibrium strategy provides at least as much payoff as in the case of agent 2 exerting no effort past state $q$, thus agent 2's equilibrium discounted payoff at state $q, J_{2}(q)$ should be strictly positive. To summarize, $J_{1}(q)>0$ and $J_{2}(q)>0$. Thus, if the project is completing at state $q$, then $J_{1}(q)$ and $J_{2}(q)$ are both strictly positive. By Lemma $3, J_{1}^{\prime}(q) \geq 0$ and $J_{2}^{\prime}(q) \geq 0$ and therefore $J_{1}$ and $J_{2}$ are strictly positive on $(q, Q)$. Equation (5) then implies that $J_{1}^{\prime}$ and $J_{2}^{\prime}$ are strictly positive on $(q, Q)$. Hence, if in some MPE the project is completing at state $q$, both agents exert strictly positive effort at all states beyond $q$ (and up to completion of the project).

The next lemma gives the values $\bar{Q}_{i}$ that are defined to be the project state that makes each agent $i$ indifferent between terminating the project at this state, and continuing the project one more instant.

Lemma 6. Assume the agents are asymmetric, i.e., $\alpha_{1} / \gamma_{1}<\alpha_{2} / \gamma_{2}$. The values of $\bar{Q}_{1}$ and $\bar{Q}_{2}$ are unique and given by

$$
\sqrt{\bar{Q}_{1}}=\frac{\sqrt{2 / 3} \sqrt{\mu} \alpha_{1} / \gamma_{1}}{\sqrt{r} \alpha_{1} / \gamma_{1}+\frac{\sqrt{r}}{12}[\sqrt{\mu}+\sqrt{3 \nu}]^{2}}
$$

and

$$
\sqrt{\bar{Q}_{2}}=\frac{\sqrt{2 / 3} \sqrt{\mu} \alpha_{2} / \gamma_{2}}{\sqrt{r} \alpha_{2} / \gamma_{2}+\frac{\sqrt{r}}{12}[\sqrt{\mu}-\sqrt{3 \nu}]^{2}}
$$


where

$$
\mu=2 \sqrt{\left(\frac{\alpha_{1}}{\gamma_{1}}\right)^{2}+\left(\frac{\alpha_{2}}{\gamma_{2}}\right)^{2}-\frac{\alpha_{1}}{\gamma_{1}} \frac{\alpha_{2}}{\gamma_{2}}}+\frac{\alpha_{1}}{\gamma_{1}}+\frac{\alpha_{2}}{\gamma_{2}}
$$

and

$$
\nu=2 \sqrt{\left(\frac{\alpha_{1}}{\gamma_{1}}\right)^{2}+\left(\frac{\alpha_{2}}{\gamma_{2}}\right)^{2}-\frac{\alpha_{1}}{\gamma_{1}} \frac{\alpha_{2}}{\gamma_{2}}}-\frac{\alpha_{1}}{\gamma_{1}}+\frac{\alpha_{2}}{\gamma_{2}} .
$$

Furthermore, $\bar{Q}_{1}<\bar{Q}_{2}$.

Proof. Consider a project of scope $Q$. Let $\bar{a}_{i}(Q)$ denote the equilibrium effort agent $i$ exerts at the very end of the project when the terminal state is $Q$. Recall that, in equilibrium, the action of agent $i$ at state $q$ is given by

$$
a_{i}(q)=J_{i}^{\prime}(q) / \gamma_{i},
$$

and thus $\bar{a}_{i}(Q)=J_{i}^{\prime}(Q) / \gamma_{i}=\widetilde{J}_{i}^{\prime}(Q)$. From Lemma 4 and noting that $\widetilde{J}_{i}(Q)=\left(\alpha_{i} / \gamma_{i}\right) Q$, we get

$$
\begin{aligned}
& \bar{a}_{1}(Q)=\sqrt{\frac{r Q}{6}}(\sqrt{\mu}+\sqrt{3 \nu}) \\
& \bar{a}_{2}(Q)=\sqrt{\frac{r Q}{6}}(\sqrt{\mu}-\sqrt{3 \nu}),
\end{aligned}
$$

with $\mu$ and $\nu$ defined as in the statement of the current lemma.

For a project of scope $Q$, agent $i$ gets value $\alpha_{i} Q$ at the completion of the project, when $q=Q$. If the project is instead of scope $Q+\Delta Q$ (for small enough $\Delta Q$ ), and if the current state is $q=Q$, there is a delay $\epsilon$ before the project is completed. To the first order in $\epsilon$, the relationship $\Delta Q=\left(\bar{a}_{1}(Q)+\bar{a}_{2}(Q)\right) \epsilon$ holds. Thus, to the first order in $\epsilon$, the net discounted value of the project to agent $i$ at state $q=Q$ is

$$
\alpha_{i}\left[Q+\left(\bar{a}_{1}(Q)+\bar{a}_{2}(Q)\right) \epsilon\right] e^{-r \epsilon}-\frac{\gamma_{i}}{2}\left(\bar{a}_{i}(Q)\right)^{2} \epsilon .
$$

At project scope $Q=\bar{Q}_{i}$, the agent is indifferent between stopping the project now (corresponding to a project scope $\bar{Q}_{i}$ ) and waiting an instant later (corresponding to a project scope $\bar{Q}_{i}+\Delta Q$ for an infinitesimal $\Delta Q$ ). So to the first order,

$$
\alpha_{i} \bar{Q}_{i}=\alpha_{i}\left(\bar{Q}_{i}+\left(\bar{a}_{1}\left(\bar{Q}_{i}\right)+\bar{a}_{2}\left(\bar{Q}_{i}\right)\right) \epsilon\right) e^{-r \epsilon}-\frac{\gamma_{i}}{2}\left(\bar{a}_{i}\left(\bar{Q}_{i}\right)\right)^{2} \epsilon .
$$

So:

$$
\alpha_{i}\left(\bar{a}_{1}\left(\bar{Q}_{i}\right)+\bar{a}_{2}\left(\bar{Q}_{i}\right)\right)-r \alpha_{i} \bar{Q}_{i}-\frac{\gamma_{i}}{2}\left(\bar{a}_{i}\left(\bar{Q}_{i}\right)\right)^{2}=0 .
$$


Solving this equation for $i=1,2$ yields

$$
\sqrt{\bar{Q}_{1}}=\frac{\sqrt{2 / 3} \sqrt{\mu} \alpha_{1} / \gamma_{1}}{\sqrt{r} \alpha_{1} / \gamma_{1}+\frac{\sqrt{r}}{12}[\sqrt{\mu}+\sqrt{3 \nu}]^{2}}
$$

and

$$
\sqrt{\bar{Q}_{2}}=\frac{\sqrt{2 / 3} \sqrt{\mu} \alpha_{2} / \gamma_{2}}{\sqrt{r} \alpha_{2} / \gamma_{2}+\frac{\sqrt{r}}{12}[\sqrt{\mu}-\sqrt{3 \nu}]^{2}}
$$

Note that

$$
\frac{\sqrt{\bar{Q}_{1}}}{\sqrt{\bar{Q}_{2}}}=\frac{12+\left(\frac{\alpha_{2}}{\gamma_{2}}\right)^{-1}[\sqrt{\mu}-\sqrt{3 \nu}]^{2}}{12+\left(\frac{\alpha_{1}}{\gamma_{1}}\right)^{-1}[\sqrt{\mu}+\sqrt{3 \nu}]^{2}} .
$$

In particular, $\bar{Q}_{1}<\bar{Q}_{2}$ if and only if the inequality

$$
\left(\frac{\alpha_{2}}{\gamma_{2}}\right)^{-1 / 2}[\sqrt{\mu}+\sqrt{3 \nu}]-\left(\frac{\alpha_{1}}{\gamma_{1}}\right)^{1 / 2}\left(\frac{\alpha_{2}}{\gamma_{2}}\right)^{-1 / 2}\left(\frac{\alpha_{2}}{\gamma_{2}}\right)^{-1 / 2}[\sqrt{\mu}-\sqrt{3 \nu}]>0
$$

holds. Let

$$
f(x)=\sqrt{1+x+2 \sqrt{1+x^{2}-x}},
$$

and

$$
g(x)=\sqrt{1-x+2 \sqrt{1+x^{2}-x}} .
$$

Note that

$$
\left(\frac{\alpha_{2}}{\gamma_{2}}\right)^{-1 / 2}[\sqrt{\mu}+\sqrt{3 \nu}]=f\left(\left(\alpha_{1} / \gamma_{1}\right)\left(\alpha_{2} / \gamma_{2}\right)^{-1}\right)+\sqrt{3} g\left(\left(\alpha_{1} / \gamma_{1}\right)\left(\alpha_{2} / \gamma_{2}\right)^{-1}\right)
$$

and that

$$
\left(\frac{\alpha_{2}}{\gamma_{2}}\right)^{-1 / 2}[\sqrt{\mu}-\sqrt{3 \nu}]=f\left(\left(\alpha_{1} / \gamma_{1}\right)\left(\alpha_{2} / \gamma_{2}\right)^{-1}\right)-\sqrt{3} g\left(\left(\alpha_{1} / \gamma_{1}\right)\left(\alpha_{2} / \gamma_{2}\right)^{-1}\right) .
$$

Since, by assumption, $\alpha_{1} / \gamma_{1}<\alpha_{2} / \gamma_{2}$, (8) is satisfied if

$$
[f(x)+\sqrt{3} g(x)]-x[f(x)-\sqrt{3} g(x)]>0
$$

for every $x \in(0,1)$. Note that, as $f, g>0$ on $(0,1)$, so

$$
\begin{aligned}
{[f(x)+\sqrt{3} g(x)]-x[f(x)-\sqrt{3} g(x)] } & \geq x[f(x)+\sqrt{3} g(x)]-x[f(x)-\sqrt{3} g(x)] \\
& \geq x[f(x)+g(x)]-x[f(x)-g(x)] \\
& =2 x g(x) \\
& >0 .
\end{aligned}
$$


This establishes the inequality (8), and thus $\bar{Q}_{1}<\bar{Q}_{2}$.

Equations (6) and (7) show that the agent's action at time of termination is strictly increasing with the project scope.

Lemma 7. The value $J_{i}^{\prime}(Q ; Q)$ is strictly increasing in $Q$. Furthermore $\bar{Q}_{i}$ is the unique solution to $J_{i}^{\prime}\left(Q_{i}(Q) ; Q_{i}(Q)\right)=\alpha_{i}$.

Proof. Consider agent $i$ 's optimization problem given state $q$. We seek the unique $q$ such that $q=\arg \max _{Q}\left\{J_{i}(q ; Q)\right\}$. For such $q$, we have $\left.\frac{\partial}{\partial Q} J_{i}(q ; Q)\right|_{q=Q}=0$. Note that $J_{i}(Q ; Q)=\alpha_{i} Q$, and totally differentiating this with respect to $Q$ yields

$$
\frac{d J_{i}(Q ; Q)}{d Q}=J_{i}^{\prime}(Q ; Q)+\left.\frac{\partial J_{i}(q ; Q)}{\partial Q}\right|_{q=Q}
$$

thus

$$
J_{i}^{\prime}(Q ; Q)=\alpha_{i}
$$

By our assumption that $J_{i}(q ; Q)$ is strictly concave in $Q$ for all $q \leq Q \leq \bar{Q}_{2}$, it follows that (9) is necessary and sufficient for a maximum.

Noting that the explicit form of the HJB equations of Lemma 4 implies that $J_{i}^{\prime}(Q ; Q)=J_{i}^{\prime}(1 ; 1) \sqrt{Q}$, it follows that $J_{i}^{\prime}(Q ; Q)$ is strictly increasing in $Q$. Therefore, the solution to (9) is unique.

\section{A.2 Proof of Proposition 1}

Existence. Fix some $Q>0$, and let $\widetilde{J}_{i}(q)=\frac{J_{i}(q)}{\gamma_{i}}$. As in Lemma 4 of Section A.1, we note that the system of ODEs of Section 3 defined by (3) subject to (2) can be rewritten as

$$
r \widetilde{J}_{i}(q)=\frac{1}{2}\left[\widetilde{J}_{i}^{\prime}(q)\right]^{2}+\widetilde{J}_{i}^{\prime}(q) \widetilde{J}_{j}^{\prime}(q)
$$

subject to $\widetilde{J}_{i}(Q)=\frac{\alpha_{i}}{\gamma_{i}} Q$ for all $i \in\{1,2\}$ and $j \neq i$. If a solution to this system of ODEs exists and $\widetilde{J}_{i}^{\prime}(q) \geq 0$ for all $i$ and $q$, then it constitutes an MPE, and each agent $i$ 's effort level satisfies $a_{i}(q)=\widetilde{J}_{i}^{\prime}(q)$.

Lemma 8. For every $\epsilon \in\left(0, \min _{i}\left\{\frac{\alpha_{i}}{\gamma_{i}} Q\right\}\right)$, there exists some $q_{\epsilon}<Q$ such that there exists a unique solution $\left(\widetilde{J}_{1}, \widetilde{J}_{2}\right)$ to the system of ODEs on $\left[q_{\epsilon}, Q\right]$ that satisfies $\widetilde{J}_{i} \geq \epsilon$ on that interval for all $i$. 
Proof. This proof follows the proof of Lemma 4 in Cvitanić and Georgiadis (2015) closely. It follows from Lemma 4 of Section A.1 that we can write (3) as

$$
\widetilde{J}_{i}^{\prime}(q)=H_{i}\left(\widetilde{J}_{1}(q), \widetilde{J}_{2}(q)\right)
$$

For given $\epsilon>0$, let

$$
M_{H}=\max _{i} \max _{\epsilon \leq x_{i} \leq \frac{\alpha_{i}}{\gamma_{i}} Q} H_{i}\left(x_{1}, x_{2}\right) .
$$

Pick $q_{\epsilon}<Q$ sufficiently large such that, for all $i$,

$$
\frac{\alpha_{i}}{\gamma_{i}} Q-\left(Q-q_{\epsilon}\right) M_{H} \geq \epsilon
$$

Then, define $\Delta q=\frac{Q-q_{\epsilon}}{N}$ and functions $\widetilde{J}_{i}^{N}$ by Euler iterations (see, for example, Atkinson et al. (2009)), going backwards from $Q$,

$$
\begin{gathered}
\widetilde{J}_{i}^{N}(Q)=\frac{\alpha_{i}}{\gamma_{i}} Q \\
\widetilde{J}_{i}^{N}(Q-\Delta q)=\frac{\alpha_{i}}{\gamma_{i}} Q-\Delta q H_{i}\left(\frac{\alpha_{1}}{\gamma_{1}} Q, \frac{\alpha_{2}}{\gamma_{2}} Q\right) \\
\widetilde{J}_{i}^{N}(Q-2 \Delta q)=J_{i}^{N}(Q-\Delta q)-\Delta q H_{i}\left(J_{1}^{N}(Q-\Delta q), \ldots, J_{n}^{N}(Q-\Delta q)\right) \\
=\frac{\alpha_{i}}{\gamma_{i}} Q-\Delta q H_{i}\left(\frac{\alpha_{1}}{\gamma_{1}} Q, \frac{\alpha_{2}}{\gamma_{2}} Q\right)-\Delta q H_{i}\left(J_{1}^{N}(Q-\Delta q), \ldots, J_{n}^{N}(Q-\Delta q)\right),
\end{gathered}
$$

and so on, until $\widetilde{J}_{i}^{N}(Q-N \Delta q)=\widetilde{J}_{i}\left(q_{\epsilon}\right)$. We then complete the definition of function $\widetilde{J}_{i}^{N}$ by making it piecewise linear between the points $Q-k \Delta q, k=1, \ldots, N$. Note from the assumption on $Q-q_{\epsilon}$ that $\widetilde{J}_{i}^{N}(Q-k \Delta q) \geq \epsilon$, for all $k=1, \ldots, N$. Since $H_{i}$ are continuously differentiable, they are Lipschitz continuous on the 2 -dimensional bounded domain $\left[\epsilon, \frac{\alpha_{1}}{\gamma_{1}} Q\right] \times\left[\epsilon, \frac{\alpha_{2}}{\gamma_{2}} Q\right]$. Therefore, by the standard ODE argument, the sequence $\left\{\widetilde{J}_{i}^{n}\right\}_{n=1}^{N}$ converges to a unique solution $\widetilde{J}_{i}$ of the system of ODEs, and we have $\widetilde{J}_{i}(q)>\epsilon$ for all $q \in\left[q_{\epsilon}, Q\right]$.

Let

$$
\underline{q}=\inf _{\epsilon>0} q_{\epsilon}
$$

Lemma 8 shows that the the system of ODEs has a unique solution on $\left[q_{\epsilon}, Q\right]$ for every $\epsilon>0$. Thus, there exists a unique solution on $(\underline{q}, Q]$. Then, by standard optimal control arguments, it follows that $\widetilde{J}_{i}(q)$ is the value function of agent $i$ for every initial project value $q>\underline{q}$. 
To establish convexity, we differentiate (5) with respect to $q$ to obtain

$$
r \widetilde{J}_{i}^{\prime}(q)=\left[\widetilde{J}_{1}^{\prime}(q)+\widetilde{J}_{2}^{\prime}(q)\right] \widetilde{J}_{i}^{\prime \prime}(q)+\widetilde{J}_{i}^{\prime}(q) \widetilde{J}_{j}^{\prime \prime}(q)
$$

or equivalently in matrix form,

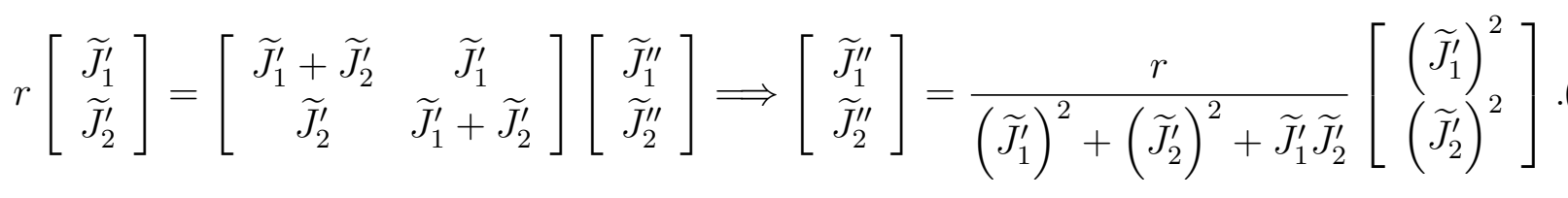

Note that $a_{i}^{\prime}(q)=\widetilde{J}_{i}^{\prime \prime}(q)>0$ if and only if $\widetilde{J}_{i}^{\prime}(q)>0$ for all $i$, or equivalently, if and only if $q>\underline{q}$.

So far, we have shown that given any $Q$, there exists some $\underline{q}<Q$ (which depends on the choice of $Q$ ) such that the system of ODEs defined by (3) subject to (2) has a project-completing solution on $(\underline{q}, Q]$. In this solution, $J_{i}(q)>0, J_{i}^{\prime}(q)>0$, and $a_{i}^{\prime}(q)>0$ for all $i$ and $q>\underline{q}$. On the other hand, Lemma 5 implies that $J_{i}(q)=J_{i}^{\prime}(q)=0$ for all $q \leq \underline{q}$. Therefore, the game starting at $q_{0}=0$ has a project-completing MPE if and only if $\underline{q}<0$.

As shown in Lemma 1 regarding the single agent case, for small enough $Q$, each agent would be exerting effort and completing the project by himself even if the other agent were to exert no effort. A fortiori, the project will complete in an equilibrium where both agents can exert effort. Hence, for $Q$ small enough, the MPE is project-completing.

As is shown in Section 3.2 regarding the socially optimal effort levels, for large enough $Q$, agents are better off not starting the project. A fortiori, for such project scopes, the project will not complete in an equilibrium where both agents can exert effort. Hence, for $Q$ large enough, the MPE is not project-completing. Instead, neither agent puts any effort on the project and the project is never started.

Uniqueness. We show that if $\left(J_{1}^{a}, J_{2}^{a}\right)$ and $\left(J_{1}^{b}, J_{2}^{b}\right)$ are two well-behaved solutions to (3) subject to the boundary constraint (2) and subject to the constraint that each of the four functions is nondecreasing, then $\left(J_{1}^{a}, J_{2}^{a}\right)=\left(J_{1}^{b}, J_{2}^{b}\right)$ on the entire range $[0, Q]$. If the value functions associated with some MPE are well-behaved, then they must satisfy (3) subject to (2), and by Lemma 3 of Section A.1 they must be nondecreasing. As the value functions uniquely pin down the equilibrium actions, it implies that for any project scope $Q$ there exists a unique MPE with well-behaved solutions to the HJB equations. 
First, consider the case $J_{1}^{a}(0)>0$. Then $J_{2}^{a}(0)>0$ by Lemma 5 of Section A.1. As $J_{1}^{a}$ and $J_{2}^{a}$ are nondecreasing, it follows from Lemma 8 that $\left(J_{1}^{a}, J_{2}^{a}\right)=\left(J_{1}^{b}, J_{2}^{b}\right)$ on the entire range $[0, Q]$. If instead $J_{1}^{b}(0)>0$, the symmetric argument applies.

Next consider the case $J_{1}^{a}(0)=J_{1}^{b}(0)=0$, and let $q^{a}=\sup \left\{q \geq 0 \mid J_{1}^{a}(q)=0\right\}$. As $J_{1}^{a}(0)=0$ we have $q^{a} \geq 0$. The boundary condition (2) and the continuity of $J_{1}$ implies that $q^{a}<Q$. Moreover, on the non-empty interval $\left(q^{a}, Q\right]$ we have $J_{1}^{a}>0$, and thus by Lemma 5 of Section A.1, $J_{1}^{b}>0$ on that same interval. Lemma 8 then implies that $\left(J_{1}^{a}, J_{2}^{a}\right)=\left(J_{1}^{b}, J_{2}^{b}\right)$ on every $\left[q^{a}+\epsilon, Q\right]$ for $\epsilon>0$, and thus that $\left(J_{1}^{a}, J_{2}^{a}\right)=\left(J_{1}^{b}, J_{2}^{b}\right)$ on $\left(q^{a}, Q\right]$. Now let us consider the range $\left[0, q^{a}\right]$. By continuity of $J_{1}^{a}$ we have $J_{1}^{a}\left(q^{a}\right)=0$. As $J_{1}^{a}$ is nondecreasing and nonnegative, then $J_{1}^{a}\left(q^{a}\right)=0$ implies that $J_{1}^{a}=0$ on the interval $\left[0, q^{a}\right]$. As $J_{1}^{a}(q)=0$ if and only if $J_{2}^{a}(q)=0$, we get that $J_{2}^{a}=0$ on the interval $\left[0, q_{0}\right]$. Thus, $\left(J_{1}^{a}, J_{2}^{a}\right)=0$ on $\left[0, q^{a}\right]$.

Similarly let $q^{b}=\sup \left\{q \mid J_{1}^{b}(q)=0\right\}$. We have $q^{b} \in[0, Q)$, and by a symmetric $\operatorname{argument}\left(J_{1}^{b}, J_{2}^{b}\right)=0$ on $\left[0, q^{b}\right]$. If $q^{b}<q^{a}$, then we get by Lemma 8 that $\left(J_{1}^{a}, J_{2}^{a}\right)=$ $\left(J_{1}^{b}, J_{2}^{b}\right)>0$ on $\left(q^{b}, Q\right]$, which contradicts $\left(J_{1}^{a}, J_{2}^{a}\right)=0$ on $\left[0, q^{a}\right]$. If instead $q^{b}>q^{a}$, then we get that $\left(J_{1}^{a}, J_{2}^{a}\right)=\left(J_{1}^{b}, J_{2}^{b}\right)>0$ on $\left(q^{a}, Q\right]$, which contradicts that $\left(J_{1}^{b}, J_{2}^{b}\right)=0$ on $\left[0, q^{b}\right]$. Hence $q^{a}=q^{b}$.

Altogether this implies that on the interval $\left[0, q^{a}\right],\left(J_{1}^{a}, J_{2}^{a}\right)=\left(J_{1}^{b}, J_{2}^{b}\right)=0$, and on the interval $\left(q^{a}, Q\right],\left(J_{1}^{a}, J_{2}^{a}\right)=\left(J_{1}^{b}, J_{2}^{b}\right)>0$. Hence the HJB equations define a unique value function and thus a unique MPE.

\section{A.3 Proof of Proposition 2}

First, we fix some $Q>0$, and we use the normalization $\widetilde{J}_{i}(q)=\frac{J_{i}(q)}{\gamma_{i}}$ as in the proof of Proposition 1.

To prove part 1 , assume that $\frac{\gamma_{1}}{\alpha_{1}}<\frac{\gamma_{2}}{\alpha_{2}}$, let $\widetilde{D}(q)=\widetilde{J}_{1}(q)-\widetilde{J}_{2}(q)$, and note that $\widetilde{D}(\cdot)$ is smooth, $\widetilde{D}(q)=0$ for $q \leq \underline{q}$ and $\widetilde{D}(Q)=\left(\frac{\alpha_{1}}{\gamma_{1}}-\frac{\alpha_{2}}{\gamma_{2}}\right) Q>0$, where $\underline{q}$ is given by (12). Observe that either $\widetilde{D}^{\prime}(q)>0$ for all $q \geq 0$, or there exists some $\bar{q} \in[0, Q]$ such that $\widetilde{D}^{\prime}(\bar{q})=0$. Suppose that the latter is the case. Then it follows from $(5)$ that $\widetilde{D}(\bar{q})=0$, which implies that $\widetilde{D}(q) \geq 0$ for all $q$, and $\widetilde{D}^{\prime}(q)>(=) 0$ if and only if $\widetilde{D}(q)>(=) 0$. Therefore, $\widetilde{D}^{\prime}(q) \geq 0$, which implies that $a_{1}(q) \geq a_{2}(q)$ for all $q \geq 0$. Observe from equation (13) in the proof of Proposition 1 , that $J_{i}^{\prime \prime}(q)=\beta \cdot\left(J_{i}^{\prime}(q)\right)^{2}$, where $\beta=r /\left[\left(\widetilde{J}_{1}^{\prime}\right)^{2}+\left(\widetilde{J_{2}^{\prime}}\right)^{2}+\widetilde{J}_{1}^{\prime} \widetilde{J}_{2}^{\prime}\right]$, and note that $a_{i}(q)=\widetilde{J}_{i}^{\prime}(q)$. Moreover, we know from part 1 of Proposition 2 that $a_{1}(q) \geq a_{2}(q)$, which implies that $J_{1}^{\prime \prime}(q) \geq J_{2}^{\prime \prime}(q)$, or 
equivalently, $a_{1}^{\prime}(q) \geq a_{2}^{\prime}(q)$ for all $q \geq 0$.

To prove part 2, note first the result for actions follows from the previous paragraph with all weak inequalities replaced with strict inequalities. Let $D(q)=\frac{J_{1}(q)}{\alpha_{1}}-\frac{J_{2}(q)}{\alpha_{2}}$, and note that $D(\cdot)$ is smooth, $D(q)=0$ for $q$ sufficiently small, and $D(Q)=0$. Therefore, either $D(q)=0$ for all $q$, or $D(\cdot)$ has an interior extreme point. Suppose that the former is true. Then for all $q$, we have $D(q)=D^{\prime}(q)=0$, which using (3) implies that

$$
r D(q)=\frac{\left[J_{1}^{\prime}(q)\right]^{2}}{2 \alpha_{1}^{2}}\left(\frac{\alpha_{2}}{\gamma_{2}}-\frac{\alpha_{1}}{\gamma_{1}}\right)=0 \Longrightarrow J_{1}^{\prime}(q)=0 .
$$

However, this is a contradiction, and so the latter must be true. Then there exists some $\bar{q}$ such that $D^{\prime}(\bar{q})=0$. Using (3) and the fact that $J_{i}^{\prime}(q) \geq 0$ for all $q$ and $J_{i}^{\prime}(q)>0$ for some $q$, this implies that $D(\bar{q}) \leq 0$. Therefore, $D(q) \leq 0$ for all $q$, which completes the proof.

Finally, if $\frac{\alpha_{1}}{\gamma_{1}}=\frac{\alpha_{2}}{\gamma_{2}}$, then it follows from the analysis above that $\widetilde{D}^{\prime}(q)=0$ and $D(q)=0$, which implies that $a_{1}(q)=a_{2}(q)$ and $\frac{J_{1}(q)}{\alpha_{1}}=\frac{J_{2}(q)}{\alpha_{2}}$ for all $q \geq 0$.

\section{A.4 Proof of Proposition 3}

To prove part 1, first suppose that $\frac{\gamma_{1}}{\alpha_{1}}=\frac{\gamma_{2}}{\alpha_{2}}$. In this case, we know from equation (4) that each agent's discounted payoff function satisfies

$$
J_{i}(q)=\frac{r \gamma_{i}}{6}\left[q-Q+\sqrt{\frac{6 \alpha_{i} Q}{r \gamma_{i}}}\right],
$$

and by maximizing $J_{i}(q)$ with respect to $Q$, we obtain that $Q_{1}(q)=Q_{2}(q)=\frac{3 \alpha_{i}}{2 r \gamma_{i}}$ for all $q$.

To prove part 2, next consider the case in which $\frac{\gamma_{1}}{\alpha_{1}}<\frac{\gamma_{2}}{\alpha_{2}}$. This part of the proof comprises 3 steps. Recall that by Lemma 6 of Section A.1, we have $\bar{Q}_{1}<\bar{Q}_{2}$.

Step 1: We show that $Q_{2}^{\prime}(q) \geq 0$ for all $q \geq \bar{Q}_{1}$.

To begin, we differentiate $\widetilde{J}_{i}(q ; Q)$ in $(5)$ with respect to $Q$ to obtain

$$
\begin{aligned}
& r \partial_{Q} \widetilde{J}_{1}(q ; Q)=\partial_{Q} a_{1}(q ; Q)\left[a_{1}(q ; Q)+a_{2}(q ; Q)\right]+a_{1}(q ; Q) \partial_{Q} a_{2}(q ; Q) \\
& r \partial_{Q} \widetilde{J}_{2}(q ; Q)=\partial_{Q} a_{2}(q ; Q)\left[a_{1}(q ; Q)+a_{2}(q ; Q)\right]+a_{2}(q ; Q) \partial_{Q} a_{1}(q ; Q)
\end{aligned}
$$

where we note $\partial_{Q} \widetilde{J}_{i}(q ; Q)=\frac{\partial}{\partial Q} \widetilde{J}_{i}(q ; Q)$, and where $\partial_{Q} a_{i}(q ; Q)=\partial_{Q} \widetilde{J}_{i}^{\prime}(q ; Q)=$ 
$\frac{\partial^{2}}{\partial Q \partial q} \widetilde{J}_{i}(q ; Q)$, and $a_{i}(q ; Q)=\widetilde{J}_{i}^{\prime}(q ; Q)=\frac{\partial}{\partial q} \widetilde{J}_{i}(q ; Q) .{ }^{28}$ Rearranging terms yields

$$
\begin{aligned}
& \frac{\left(a_{1}+a_{2}\right)^{2}-a_{1} a_{2}}{r}\left(\partial_{Q} a_{1}\right)=\left(a_{1}+a_{2}\right)\left(\partial_{Q} \widetilde{J}_{1}\right)-a_{1}\left(\partial_{Q} \widetilde{J}_{2}\right) \\
& \frac{\left(a_{1}-a_{2}\right)^{2}+a_{1} a_{2}}{r}\left(\partial_{Q} a_{2}\right)=\left(a_{1}+a_{2}\right)\left(\partial_{Q} \widetilde{J}_{2}\right)-a_{2}\left(\partial_{Q} \widetilde{J}_{1}\right),
\end{aligned}
$$

where we drop the arguments $q$ and $Q$ for notational simplicity. Because $a_{i}, a_{j}>0$, note that $\left(a_{1}+a_{2}\right)^{2}-a_{1} a_{2}>0$ and $\left(a_{1}-a_{2}\right)^{2}+a_{1} a_{2}>0$. Recall $Q_{i}(q)$ is agent $i$ 's ideal project scope given the current state $q$. Then for all $q<Q_{i}(q)$ and for the smallest $q$ such that $q=Q_{i}(q)$, we have $\frac{\partial}{\partial Q} \widetilde{J}_{i}\left(q ; Q_{i}(q)\right)=0$. Differentiating this with respect to $q$ yields

$$
\frac{\partial^{2}}{\partial Q \partial q} \widetilde{J}_{i}\left(q ; Q_{i}(q)\right)+\frac{\partial^{2}}{\partial Q^{2}} \widetilde{J}_{i}\left(q ; Q_{i}(q)\right) Q_{i}^{\prime}(q)=0 \Longrightarrow Q_{i}^{\prime}(q)=-\frac{\partial_{Q} a_{i}\left(q ; Q_{i}(q)\right)}{\partial_{Q}^{2} \widetilde{J}_{i}\left(q ; Q_{i}(q)\right)}
$$

Since $\partial_{Q}^{2} \widetilde{J}_{i}(q ; Q)<0$ (by our strict concavity assumption), it follows that $Q_{i}^{\prime}(q) \leq 0$ if and only if $\partial_{Q} a_{i}(q ; Q) \geq 0$.

Next, fix some $\widehat{q} \in\left(\bar{Q}_{1}, \bar{Q}_{2}\right)$. By the strict concavity of $\widetilde{J}_{i}(q ; Q)$ in $Q$, it follows that $\partial_{Q} \widetilde{J}_{1}\left(\widehat{q}, Q_{2}(\widehat{q})\right)<0$ and $\partial_{Q} \widetilde{J}_{2}\left(\widehat{q}, Q_{2}(\widehat{q})\right)=0$; i.e., agent 1 would prefer to have completed the project at a smaller project scope than $Q_{2}(\widehat{q})$, whereas agent 2 finds it optimal to complete the project at $Q_{2}(\widehat{q})$ (the latter statement being true by definition of $\left.Q_{2}(\widehat{q})\right)$. Using (15) it follows that $\partial_{Q} a_{2}\left(\widehat{q}, Q_{2}(\widehat{q})\right)>0$, which implies that $Q_{2}^{\prime}(\widehat{q})>0$. Therefore, $Q_{2}^{\prime}(q)>0$ for all $q \in\left(\bar{Q}_{1}, \bar{Q}_{2}\right)$ and $Q_{2}\left(\bar{Q}_{1}\right)>\bar{Q}_{1}$, where the last inequality follows from the facts that by assumption $\widetilde{J}_{2}(q ; Q)$ is strictly concave in $Q$ for $q \leq Q \leq \bar{Q}_{2}$ and so it admits a unique maximum, and that $\widetilde{J}_{2}^{\prime}\left(\bar{Q}_{1} ; \bar{Q}_{1}\right)<\frac{\alpha_{2}}{\gamma_{2}}$, which implies that he prefers to continue work on the project rather than complete it at $\bar{Q}_{1}$.

Step 2: $\quad$ We show that $Q_{1}^{\prime}(q) \leq 0 \leq Q_{2}^{\prime}(q)$ for all $q \leq \bar{Q}_{1}$. Moreover, $Q_{1}^{\prime}(q)<0<$ $Q_{2}^{\prime}(q)$ for all $q$ such that $Q_{1}(q)<Q_{2}(q)$.

Because $Q_{2}\left(\bar{Q}_{1}\right)>\bar{Q}_{1}$ and $Q_{i}(\cdot)$ is smooth, there exists some $\bar{q} \geq 0$ such that $Q_{2}(q)>Q_{1}(q)$ for all $q \in\left(\bar{q}, \bar{Q}_{1}\right)$. Pick some $q$ in this interval, and note that $\partial_{Q} \widetilde{J}_{1}\left(q, Q_{2}(q)\right)<0$ and $\partial_{Q} \widetilde{J}_{2}\left(q, Q_{2}(q)\right)=0$, which together with (15) implies that $\partial_{Q} a_{2}\left(q, Q_{2}(q)\right)>0$. Similarly, we have $\partial_{Q} \widetilde{J}_{1}\left(q, Q_{1}(q)\right)=0$ and $\partial_{Q} \widetilde{J}_{2}\left(q, Q_{1}(q)\right)>0$, which together with $(14)$ implies that $\partial_{Q} a_{1}\left(q, Q_{1}(q)\right)<0$. Therefore, $Q_{1}^{\prime}(q)<0<$

\footnotetext{
${ }^{28}$ Note $a_{i}(q ; Q)$ is distinct from agent strategies in the case of commitment $a_{i}(q, Q)$. Here $a_{i}(q ; Q)$ denotes agents' actions in the MPE with exogenous project scope $Q$.
} 
$Q_{2}^{\prime}(q)$ for all $q \in\left(\bar{q}, \bar{Q}_{1}\right)$.

Next, by way of contradiction, assume that there exists some $q$ such that $Q_{1}(q)>$ $Q_{2}(q)$ for some $q<\bar{q}$. Because $Q_{i}(q)$ is smooth, by the intermediate value theorem, there exists some $\widetilde{q}$ such that $Q_{1}(\widetilde{q})>Q_{2}(\widetilde{q})$ and at least one of the following statements is true: $Q_{1}^{\prime}(\widetilde{q})<0$ or $Q_{2}^{\prime}(\widetilde{q})>0$. This implies that for such $\widetilde{q}$, we must have $\partial_{Q} \widetilde{J}_{1}\left(\widetilde{q}, Q_{2}(\widetilde{q})\right)>0, \partial_{Q} \widetilde{J}_{2}\left(\widetilde{q}, Q_{2}(\widetilde{q})\right)=0, \partial_{Q} \widetilde{J}_{1}\left(\widetilde{q}, Q_{1}(\widetilde{q})\right)=0$ and $\partial_{Q} \widetilde{J}_{2}\left(\widetilde{q}, Q_{1}(\widetilde{q})\right)<0$. Then it follows from (14) and (15) that $\partial_{Q} a_{1}\left(\widetilde{q}, Q_{2}(\widetilde{q})\right)>0$ and $\partial_{Q} a_{2}\left(\widetilde{q}, Q_{1}(\widetilde{q})\right)<0$. This in turn implies that $Q_{1}^{\prime}(\widetilde{q})>0>Q_{2}^{\prime}(\widetilde{q})$, which is a contradiction. Therefore, it must be the case that $Q_{2}(q) \geq Q_{1}(q)$ for all $q$, and therefore $Q_{1}^{\prime}(q) \leq 0$ for all $q \leq \bar{Q}_{1}$ and $Q_{2}^{\prime}(q) \geq 0$ for all $q \leq \bar{Q}_{2}$.

Step 3: We show that there does not exist any $q$ such that $Q_{1}(q)=Q_{2}(q)$.

First, we show that if there exists some $\bar{q}$ such that $Q_{1}(\bar{q})=Q_{2}(\bar{q})$, then it must be the case that $Q_{1}(q)=Q_{2}(q)$ for all $q \leq \bar{q}$. Suppose that the converse is true. Then by the intermediate value theorem, there exists some $\widetilde{q}$ such that $Q_{1}(\widetilde{q})<Q_{2}(\widetilde{q})$ and at least one of the following statements is true: either $Q_{1}^{\prime}(\widetilde{q})>0$ or $Q_{2}^{\prime}(\widetilde{q})<0$. This implies that for such $\widetilde{q}$, we must have $\partial_{Q} \widetilde{J}_{1}\left(\widetilde{q}, Q_{2}(\widetilde{q})\right)<0, \partial_{Q} \widetilde{J}_{2}\left(\widetilde{q}, Q_{2}(\widetilde{q})\right)=0$, $\partial_{Q} \widetilde{J}_{1}\left(\widetilde{q}, Q_{1}(\widetilde{q})\right)=0$ and $\partial_{Q} \widetilde{J}_{2}\left(\widetilde{q}, Q_{1}(\widetilde{q})\right)>0$. Then it follows from (14) and (15) that $\partial_{Q} a_{1}\left(\widetilde{q}, Q_{2}(\widetilde{q})\right)<0$ and $\partial_{Q} a_{2}\left(\widetilde{q}, Q_{1}(\widetilde{q})\right)>0$. This in turn implies that $Q_{1}^{\prime}(\widetilde{q})<$ $0<Q_{2}^{\prime}(\widetilde{q})$, which is a contradiction. Therefore, if there exists some $\bar{q}$ such that $Q_{1}(\bar{q})=Q_{2}(\bar{q})$, then $Q_{1}(q)=Q_{2}(q)$ and $\partial_{Q} a_{1}(q ; Q)=\partial_{Q} a_{2}(q ; Q)=0$ for all $q \leq \bar{q}$ and $Q=Q_{1}(q)$.

Next, note that each agent's normalized discounted payoff function can be written in integral form as

$$
\widetilde{J}_{i}\left(q_{t} ; Q\right)=e^{-r[\tau(Q)-t]} \frac{\alpha_{i}}{\gamma_{i}} Q-\int_{t}^{\tau(Q)} e^{-r(s-t)} \frac{\left(a_{i}\left(q_{s} ; Q\right)\right)^{2}}{2} d s .
$$

Differentiating this with respect to $Q$ yields the first-order condition $e^{-r[\tau(Q)-t]} \frac{\alpha_{i}}{\gamma_{i}}\left[1-r Q \tau^{\prime}(Q)\right]-e^{-r[\tau(Q)-t]} \tau^{\prime}(Q) \frac{\left(a_{i}(Q ; Q)\right)^{2}}{2}-\int_{t}^{\tau(Q)} e^{-r(s-t)} a_{i}\left(q_{s} ; Q\right) \partial_{Q} a_{i}\left(q_{s} ; Q\right) d s=0$.

Now, by way of contradiction, suppose there exists some $\bar{q}$ such that $Q_{1}(\bar{q})=$ $Q_{2}(\bar{q})=Q^{*}$. Then we have $Q_{1}(q)=Q_{2}(q)$ and $\partial_{Q} a_{1}\left(q ; Q^{*}\right)=\partial_{Q} a_{2}\left(q ; Q^{*}\right)=0$ for 
all $q \leq \bar{q}$. Therefore, fixing some $q \leq \bar{q}$ and $Q^{*}=Q_{1}(\bar{q})$, it follows from (16) that

$$
2\left[1-r Q^{*} \tau^{\prime}\left(Q^{*}\right)\right]=\tau^{\prime}\left(Q^{*}\right) \frac{\gamma_{1}}{\alpha_{1}}\left(a_{1}\left(Q^{*} ; Q^{*}\right)\right)^{2}=\tau^{\prime}\left(Q^{*}\right) \frac{\gamma_{2}}{\alpha_{2}}\left(a_{2}\left(Q^{*} ; Q^{*}\right)\right)^{2} .
$$

Observe that $\partial_{Q} a_{1}\left(q ; Q^{*}\right)=\partial_{Q} a_{2}\left(q ; Q^{*}\right)=0$, which implies that $\partial_{Q}\left[a_{1}\left(q ; Q^{*}\right)+a_{2}\left(q ; Q^{*}\right)\right]=$ 0 , and hence $\tau^{\prime}\left(Q^{*}\right)>0$. By assumption, $\frac{\gamma_{1}}{\alpha_{1}}<\frac{\gamma_{2}}{\alpha_{2}}$, and we shall now show that $\frac{\gamma_{1}}{\alpha_{1}}\left(a_{1}\left(Q^{*} ; Q^{*}\right)\right)^{2}>\frac{\gamma_{2}}{\alpha_{2}}\left(a_{2}\left(Q^{*} ; Q^{*}\right)\right)^{2}$. Let $D\left(q ; Q^{*}\right)=\sqrt{\frac{\gamma_{1}}{\alpha_{1}}} \widetilde{J}_{1}\left(q ; Q^{*}\right)-\sqrt{\frac{\gamma_{2}}{\alpha_{2}}} \widetilde{J}_{2}\left(q ; Q^{*}\right)$, and note that $D\left(q ; Q^{*}\right)=0$ for $q$ sufficiently small, $D\left(Q^{*} ; Q^{*}\right)=\left(\sqrt{\frac{\alpha_{1}}{\gamma_{1}}}-\sqrt{\frac{\alpha_{2}}{\gamma_{2}}}\right) Q^{*}>$ 0 , and $D\left(\cdot ; Q^{*}\right)$ is smooth. Therefore, either $D^{\prime}\left(q ; Q^{*}\right)>0$ for all $q$, or there exists some extreme point $z$ such that $D^{\prime}\left(z ; Q^{*}\right)=0$. If the former is true, then $D^{\prime}\left(Q^{*} ; Q^{*}\right)>0$, and we obtain the desired result. Now suppose that the latter is true. It follows from (5) that

$$
r D\left(z ; Q^{*}\right)=\frac{\left[\widetilde{J_{1}^{\prime}}\left(z ; Q^{*}\right)\right]^{2}}{2}\left(\sqrt{\frac{\gamma_{1}}{\alpha_{1}} \frac{\alpha_{2}}{\gamma_{2}}}-1\right)<0,
$$

which implies that any extreme point $z$ must satisfy $D\left(z ; Q^{*}\right)<0<D\left(Q^{*} ; Q^{*}\right)$, and hence $D^{\prime}\left(Q^{*} ; Q^{*}\right)>0$. Therefore, $\frac{\gamma_{1}}{\alpha_{1}}\left(a_{1}\left(Q^{*} ; Q^{*}\right)\right)^{2}>\frac{\gamma_{2}}{\alpha_{2}}\left(a_{2}\left(Q^{*} ; Q^{*}\right)\right)^{2}$, which contradicts the assumption that there exists some $q$ such that $Q_{1}(q)=Q_{2}(q)$.

We complete the proof of Proposition 3. From Lemma 6 of Section A.1, we know that $\bar{Q}_{1}<\bar{Q}_{2}$. Steps 1 and 2 show that $Q_{1}^{\prime}(q) \leq 0$ for all $q \leq \bar{Q}_{1}$ and $Q_{2}^{\prime}(q) \geq 0$ for all $q \leq \bar{Q}_{2}$, respectively, while step 3 shows that there exists no $q<\bar{Q}_{2}$ such that $Q_{1}(q)=Q_{2}(q)$. This proves part 2(a). To see part 2(b), Step 3 shows that $Q_{2}(q)>Q_{1}(q)$ for all $q$ (i.e. $\bar{q}=0$ ), which together with Step 2, implies that $Q_{2}^{\prime}(q)>0>Q_{1}^{\prime}(q)$ for all $q>0$. Finally, it follows from the strict concavity of $J_{i}(q ; Q)$ in $Q$ that $Q_{i}(q)=q$ for all $q \geq \bar{Q}_{i}$, which completes the proof of part $2(\mathrm{c})$.

\section{A.5 Proof of Lemma 1}

First, we characterize each agent $i$ 's effort and payoff function when he works alone on the project (and receives $\alpha_{i} Q$ upon completion).

Let $\widehat{J}_{i}(q ; Q)$ be agent $i$ 's discounted payoff at state $q$ for a project of scope $Q$. By standard arguments, under regularity conditions, the function $\widehat{J}_{i}(\cdot ; Q)$ satisfies the HJB equation

$$
r \widehat{J}_{i}(q ; Q)=\max _{\check{a}_{i}}\left\{-\frac{\gamma}{2} \check{a}_{i}^{2}+\check{a}_{i} \widehat{J}_{i}^{\prime}(q ; Q)\right\}
$$


subject to the boundary condition

$$
\widehat{J}_{i}(Q ; Q)=\alpha_{i} Q
$$

The game defined by (17) subject to the boundary condition (18) has a unique solution on $(\underline{q}, Q]$ in which the project is completed, where $\underline{q}=Q-\sqrt{\frac{2 \alpha_{i} Q}{r \gamma_{i}}}$. Then agent $i$ 's effort strategy and discounted payoff satisfies

$$
\begin{aligned}
\widehat{a}_{i}(q ; Q) & =r\left(q-Q+\sqrt{\frac{2 \alpha_{i} Q}{r \gamma_{i}}}\right) \\
\text { and } \widehat{J}_{i}(q ; Q) & =\frac{r \gamma_{i}}{2}\left(q-Q+\sqrt{\frac{2 \alpha_{i} Q}{r \gamma_{i}}}\right)^{2},
\end{aligned}
$$

respectively. Define

$$
\widehat{Q}_{i}(q)=\arg \max _{Q \geq q}\left\{\widehat{J}_{i}(q ; Q)\right\}
$$

It is straightforward to verify that $\widehat{Q}_{i}(q)=\frac{\alpha_{i}}{2 r \gamma_{i}}$. The inequality $\widehat{Q}_{2}(q)<\widehat{Q}_{1}(q)$ follows from the fact that by assumption $\frac{\gamma_{1}}{\alpha_{1}}<\frac{\gamma_{2}}{\alpha_{2}}$.

Next, we show that $\widehat{Q}_{1}(q)<\bar{Q}_{1}$. Define $\widehat{\Delta}(q)=J_{1}\left(q ; \bar{Q}_{1}\right)-\widehat{J}_{1}\left(q ; \bar{Q}_{1}\right)$. Note that $J_{1}^{\prime}\left(\bar{Q}_{1} ; \bar{Q}_{1}\right)=\alpha_{1}, \widehat{\Delta}\left(\bar{Q}_{1}\right)=0, \widehat{\Delta}(q)=0$ for sufficiently small $q$, and $\widehat{\Delta}(\cdot)$ is smooth. Therefore, either $\widehat{\Delta}(q)=0$ for all $q$, or it has an interior local extreme point. In either case, there exists some $z$ such that $\widehat{\Delta}^{\prime}(z)=0$. Using (3) and the fact that, from the single agent HJB equation, $r \widehat{J}_{1}(q ; Q)=\left[\widehat{J}_{1}^{\prime}(q ; Q)\right]^{2} /\left(2 \gamma_{1}\right)$, it follows that

$$
r \widehat{\Delta}(z)=\frac{J_{1}^{\prime}\left(z ; \bar{Q}_{1}\right) J_{2}^{\prime}\left(z ; \bar{Q}_{1}\right)}{\gamma_{2}} .
$$

Because $J_{1}^{\prime}\left(q ; \bar{Q}_{1}\right) J_{2}^{\prime}\left(q ; \bar{Q}_{1}\right)>0$ for at least some $q$, it follows that it cannot be the case that $\widehat{\Delta}(q)=0$ for all $q$. Because $J_{1}^{\prime}\left(q ; \bar{Q}_{1}\right) J_{2}^{\prime}\left(q ; \bar{Q}_{1}\right) \geq 0$, it follows that any extreme point $z$ must satisfy $\widehat{\Delta}(z) \geq 0$, which together with the boundary conditions implies that $\widehat{\Delta}(q) \geq 0$ for all $q$. Therefore, $\widehat{\Delta}^{\prime}\left(\bar{Q}_{1}\right)<0$, which in turn implies that $\widehat{J}_{1}^{\prime}\left(\bar{Q}_{1} ; \bar{Q}_{1}\right)>J_{1}^{\prime}\left(\bar{Q}_{1} ; \bar{Q}_{1}\right)=\alpha_{1}$. By noting that $\widehat{J}_{1}^{\prime}\left(\widehat{Q}_{1}(q) ; \widehat{Q}_{1}(q)\right)=\alpha_{1}$ and $\widehat{J}_{1}^{\prime}(Q ; Q)$ is strictly increasing in $Q$, it follows that $\widehat{Q}_{1}(q)<\bar{Q}_{1}$.

Since $Q_{1}^{\prime}(q)<0$ for all $q$, it follows that $\widehat{Q}_{1}(q)<Q_{1}(q)$ for all $q$, and we know from Proposition 3 that $Q_{1}(q)<Q_{2}(q)$ for all $q$. 


\section{A.6 Proof of Lemma 2}

Let $S(q ; Q)=J_{1}(q ; Q)+J_{2}(q ; Q)$. Because $J_{i}(q ; Q)$ is strictly concave in $Q$ for all $i$ and $q \leq Q \leq \bar{Q}_{2}$, it follows that $S(q ; Q)$ is also strictly concave in $Q$ for all $q \leq Q \leq \bar{Q}_{2}$. Therefore, $Q^{*}(q)$ will satisfy $\frac{\partial}{\partial Q} S(q ; Q)=0$ at $Q=Q^{*}(q)$ and $\frac{\partial}{\partial Q} S(q ; Q)$ is strictly decreasing in $Q$ for all $q$. We know from Proposition 3 that $Q_{1}(q)<Q_{2}(q)$ for all $q \leq \bar{Q}_{2}$. Moreover, we know that (i) $\frac{\partial}{\partial Q} J_{1}(q ; Q) \geq 0$ and $\frac{\partial}{\partial Q} J_{2}(q ; Q)>0$ and so $\frac{\partial}{\partial Q} S(q ; Q)>0$ for all $q \leq Q_{1}(q)$, and (ii) $\frac{\partial}{\partial Q} J_{1}(q ; Q)<0$ and $\frac{\partial}{\partial Q} J_{2}(q ; Q) \leq 0$ and so $\frac{\partial}{\partial Q} S(q ; Q)<0$ for all $q \geq Q_{2}(q)$. Because $\frac{\partial}{\partial Q} S(q ; Q)$ is strictly decreasing in $Q$, it follows that $\frac{\partial}{\partial Q} S(q ; Q)=0$ for some $Q \in\left(Q_{1}(q), Q_{2}(q)\right)$.

\section{A.7 Proof of Proposition 4}

We first construct a project-completing MPE with project scope $Q_{i}(0)$.

Consider the following strategy profile.

- Effort levels: let both agents exert no effort at all states before the project scope has been decided. Once a project scope $Q$ has been decided, let both agents choose their respective effort level in the benchmark setting of Section 3 for a project of exogenous scope $Q$ at all states $q \leq Q$, and let them exert no effort for all states $q>Q$.

- Dictator's decision: any state $q$ where no scope has yet been decided, let the dictator set the project scope $Q_{i}(q)$.

We verify that such strategy profile is an MPE.

First, let us fix the strategy of the dictator as given. Then at any state $q$, if the dictator's decision is yet to be made, agent $j$ anticipates the scope to be set immediately and is then indifferent between all effort levels. Exerting no effort is thus a best response. At any state $q$, if a decision of scope $Q$ has been made by the dictator, agent $j$ 's effort levels are, by definition, a best response to the dictator's effort strategy.

Second, let us fix the effort strategy of agent $j$. If, at state $q$, the project scope has not been decided, the dictator never profits by delaying the decision to commit because agent $j$ exerts no effort before the project scope is decided. Therefore, it is a best response to commit at state $q$. Furthermore, if he commits to project scope $Q \neq Q_{i}(q)$, the dictator's discounted payoff is $J_{i}(q ; Q) \leq J_{i}\left(q ; Q_{i}(q)\right)$. Hence commiting at state 
$q$ to project scope $Q_{i}(q)$ is a best response. The effort levels of the dictator are, by definition, a best response to agent $j$ 's strategy.

Finally, we note that in any MPE - surplus maximizing or not - the dictator commits at the beginning of the project. Suppose he was to commit after the project started, say when the project reaches state $\check{q}>0$. Since $J_{i}(\check{q} ; Q)$ has a unique maximum in $Q$, he commits to $Q_{i}(\check{q})$ and obtains payoff $J_{i}\left(\cdot ; Q_{i}(\check{q})\right)$. Then at state $q=0$ there is a profitable deviation to commit immediately to $Q_{i}(0)$ and obtain payoff $J_{i}\left(0 ; Q_{i}(0)\right)>J_{i}\left(0 ; Q_{i}(\check{q})\right)$. Hence there is no MPE in which the dictator delays the announcement of the project scope.

\section{A.8 Proof of Proposition 5}

We begin by showing that if a project-completing equilibrium exists and has scope $Q$, and if agent $i$ is dictator, then $Q \leq \bar{Q}_{i}$.

In an equilibrium of project scope $Q$, both agents anticipate that the project will be completed at state $Q$. Therefore they will both work as they would in a the benchmark game of fixed project scope $Q$ described in Section 3. In particular, at any state $q \in[0, Q]$, each agent $k \in\{1,2\}$ gets continuation payoff $J_{k}(q ; Q)$.

If $Q>\bar{Q}_{i}$, then taking any state $q \in\left(\bar{Q}_{i}, Q\right)$, Proposition 3 implies $J_{i}(q ; q)>$ $J_{i}(q ; Q)$, i.e., the dictator is strictly better off stopping the project when at state $q$, instead of stopping at state $Q$. Thus $Q \leq \bar{Q}_{i}$ in equilibrium.

Next, we show that, if agent 1 is the dictator, then $Q=\bar{Q}_{1}$ can be sustained in an MPE, whereas if agent 2 is the dictator, then $Q=Q^{*}(0)$ can be sustained in an MPE. Observe that the latter project scope maximizes total surplus among all project scopes, while the former project scope maximizes total surplus among all project scopes $Q$ that satisfy the constraint $Q \leq \bar{Q}_{1}$. Thus the MPE we will obtain satisfies the surplus-maximizing refinement used thoughout this section.

Let $Q=\bar{Q}_{1}$ if agent 1 is the dictator and let $Q=Q^{*}(0)$ if agent 2 is the dictator. Consider the following strategy profile:

- Effort levels: for any state $q \leq Q$, let both agents choose their effort optimally in a game of fixed project scope $Q$, and for all $q>Q$ let them exert no effort. Note that the unique MPE of a project of fixed scope $Q$ is completing, so both agents put positive effort at every state up to $Q \cdot{ }^{29} J_{k}(q, Q)>0$ for every $Q<Q^{*}(0)$-in

\footnotetext{
${ }^{29}$ Note that the project is completing at scope $Q^{*}(0)$. Thus for any $q<Q^{*}(0)$ and $k \in\{1,2\}$,
} 
particular for $Q=\bar{Q}_{1}$.

- Dictator's decision: let the dictator stop the project immediately whenever $q \geq Q$.

To show such strategy profile is an MPE, we must show that agents play a best response to each other at every state.

First, take the dictator's strategy as given. Then agent $j$ anticipates to be working on a project of scope $Q$, and it follows directly from the agent $j$ 's effort strategy that agent $j$ plays a best response at every state $q \leq Q$. Besides at any state $q>Q$ agent $j$ anticipates that the dictator completes the project immediately and so is indifferent between all effort levels - in particular, putting no effort is a best response.

We note that given $Q$, the effort strategy for both agents is the effort chosen in the unique MPE of the game with fixed project scope $Q$. Therefore, if the dictator follows the conjectured equilibrium strategy, then agent $j$ 's effort strategy for states up to $Q$ maximizes his payoff given the effort strategy of agent $i$, so agent $j$ has no incentive to deviate from the conjectured effort strategy. For states $q>Q$, agent $j$ is indifferent among all effort levels, in anticipation that agent $i$ completes the project immediately, and so once again has no incentive to deviate. Similarly, given $Q$, agent $i$ 's effort strategy maximizes his payoff given the conjectured effort strategy of agent $j$.

Now let's take agent $j$ 's strategy as given. If the dictator completes the project at state $Q$, then the dictator's effort level is optimal given $j$ 's effort level, by definition of agent $i$ 's effort strategy.

Let us check that terminating the project at every state $q \geq Q$ is optimal for the dictator. Consider state $q \geq Q$. As agent $j$ exerts no effort for all states greater that $Q$, and as $Q \geq \bar{Q}_{1}>\widehat{Q}_{i}$, the dictator has no incentives to continue the project by himself: he is always best off stopping the project immediately.

Now consider state $q<Q$.

- If agent 1 is the dictator then as $q<\bar{Q}_{1}<Q_{1}(q)$, by our assumption that $J_{1}(q ; Q)$ is strictly concave on $\left[q, \bar{Q}_{2}\right]$ and as $J_{1}(q ; Q)$ is maximized in $Q$ for $Q=Q_{1}(q)$, then $J_{1}(q ; Q)$ is strictly increasing in $Q$ on $\left[q, Q_{1}(q)\right]$. This implies $J_{1}\left(q ; Q_{1}(q)\right)>J_{1}\left(q ; \bar{Q}_{1}\right)>J_{1}(q ; q)$, and so the agent has no incentive to collect the termination payoff before reaching state $\bar{Q}_{1}$.

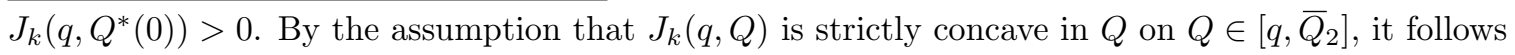
that 
- If agent 2 is the dictator then by Lemma 7 of Section A.1, $J_{2}^{\prime}(Q ; Q)$ increases in $Q$, and $J_{2}^{\prime}\left(\bar{Q}_{2} ; \bar{Q}_{2}\right)=J_{2}^{\prime}\left(Q_{2}\left(\bar{Q}_{2}\right) ; Q_{2}\left(\bar{Q}_{2}\right)\right)=\alpha_{2}$. Besides, $J_{2}(Q ; Q)=\alpha_{2} Q$ and Proposition 1 shows that $J_{2}(q ; Q)$ is strictly convex in $q$ for $q \leq Q$. Hence $J_{2}^{\prime}(q ; Q)<\alpha_{2}$ for $q<Q<\bar{Q}_{2}$, which in turn implies that $J_{2}(q ; Q)>\alpha_{2} q$ for all $q<Q$ with $Q<\bar{Q}_{2}$. Hence $J_{2}(q ; q)=\alpha_{2} q<J_{2}\left(q ; Q^{*}(0)\right)$, and thus agent 2 has no incentive to complete the project before reaching state $Q^{*}(0)$.

In conclusion, the strategies defined above form a project-completing MPE with project scope $Q$.

\section{A.9 Proof of Proposition 6}

We construct a project-completing MPE with ex-ante surplus-maximizing project scope $Q^{*}(0)$.

To do so, we consider the following strategy profile, and prove it is an equilibrium.

- Effort levels: for both agents 1 and 2 , and for every $Q \geq 0$ and $q \leq Q$, let $a_{1}(q ; Q)$ and $a_{2}(q ; Q)$ be the effort level of their respective equilibrium strategies in the benchmark setting of Section 3 for a project of exogenous scope $Q$. For $q>Q$, let $a_{1}(q, Q)=a_{2}(q, Q)=0$. Similarly, for $q \leq Q^{*}(0)$, let $a_{1}(q ;-1)$ and $a_{2}(q ;-1)$ be the effort level of their respective equilibrium strategies in the benchmark setting of Section 3 for a project of exogenous scope $Q^{*}(0)$. For $q>Q^{*}(0)$, let $a_{1}(q,-1)=a_{2}(q,-1)=0$.

- Agenda setter proposals: let the agenda setter propose project scope $Q^{*}(0)$ at every state $q \leq Q^{*}(0)$, and propose to stop the project immediately at every state $q>Q^{*}(0)$.

- Agent $j$ 's decisions: in a project state $q>Q^{*}(0)$, agent $j$ accepts the agenda setter's proposal to stop at $Q$ for all $Q$ with $J_{j}(q ; Q) \geq J_{j}(q ; q)$, and rejects the proposal otherwise. In a state $q \leq Q^{*}(0)$, let agent $j$ accept the agenda setter's proposal to stop at $Q$ whenever $J_{j}(q ; Q) \geq J_{j}\left(q ; Q^{*}(0)\right)$ and reject the proposal otherwise.

To show such strategy profile is an MPE, we must show that agents play a best response to each other at every state. 
First, take the agenda setter's strategy as given. Then it follows directly from agent $j$ 's strategy that agent $j$ plays a best response at every state - both in terms of effort and response to proposals of the agenda setter.

Now take the strategy of agent $j$ as given. If at state $q$ a project scope $Q$ has already been agreed upon, the agenda setter, who can no longer change the project scope, plays a best response (in terms of effort level) to the strategy of agent $j$. It remains to show that the agenda setter plays a best response at every $q$ when no project state has been agreed on yet. If he anticipates the project scope to be $Q^{*}(0)$, his effort levels are optimal in every state. Let us check that the proposal strategy is indeed optimal, and yield project scope $Q^{*}(0)$.

- If $q \geq Q^{*}(0)$, and agent 1 is the agenda setter, then agent 1 is better off if the project stops immediately: since $Q_{1}(q)=q, J_{1}(q ; q)>J_{1}(q ; Q)$ for every $Q>q$. If agent 1 proposes to stop the project at state $q$, then agent 2 accepts, by definition of agent 2's strategy. Hence it is optimal for agent 1 to propose to stop the project at state $q$, and the conjectured equilibrium strategy of agent 1 is a best response to agent 2's strategy.

- If $q \geq Q^{*}(0)$, and agent 2 is the agenda setter, then agent 2 would prefer in some cases to pursue the project with agent 1 , but never wants to pursue the project by himself. As agent 1 only accepts proposals to stop right away, and as he exerts no effort past state $Q^{*}(0)$ until a scope proposed is accepted, agent 2 is better off proposing to stop the project at the current state $q$-proposition accepted by agent 1 . Hence the conjectured equilibrium strategy of agent 2 is a best response to agent 1's strategy.

- If $q<Q^{*}(0)$, and agent 1 is the agenda setter, then the agenda setter can guarantee himself a continuation payoff $J_{i}\left(q ; Q^{*}(0)\right)$ by following the strategy defined in the above conjectured equilibrium profile. Assume by contradiction that there is an alternative strategy for the agenda setter that yields a strictly higher payoff. Such strategy must generate a different project scope, $Q$. In addition, that project scope must be less than $Q^{*}(0)$ for agent 1 to be better off, and so an agreement must be reached before state $Q^{*}(0)$. But then $J_{2}(q ; Q)<$ $J_{2}\left(q ; Q^{*}(0)\right)$, and by definition of agent 2's strategy, agent 2 would not accept agent 1's proposal to set scope $Q$ at any state $q<Q^{*}(0)$. Hence the conjectured equilibrium strategy of agent 1 is a best response to agent 2's strategy. 
- If $q<Q^{*}(0)$, and agent 2 is the agenda setter, then as before the agenda setter can guarantee himself a continuation payoff $J_{2}\left(q ; Q^{*}(0)\right)$ by following the strategy defined in the above conjectured equilibrium profile. Assume by contradiction that there is an alternative strategy for the agenda setter that yields strictly higher payoff with a different project scope $Q$. Then, as agent 2 is strictly better off, it must be that $Q>Q^{*}(0)$, as $J_{2}(q ; Q)$ is strictly increasing in $Q$ when $Q<Q^{*}(0)$. However, agent 1 would not accept such a proposal of project $Q$ before reaching state $Q^{*}(0)$. He may accept such a proposal in state $q=Q$, however, between state $Q^{*}(0)$ and $Q$ exerts no effort. As $Q^{*}(0)>\widehat{Q}_{2}$, agent 2 is never better off pursuing and completing the project by himself past state $Q^{*}(0)$, and thus a project scope $Q=Q^{*}(0)$ is optimal. Hence the conjectured equilibrium strategy of agent 2 is a best response to agent 1's strategy.

Therefore the conjectured strategy profile constitutes a project-completing MPE with project scope $Q^{*}(0)$.

\section{A.10 Proof of Proposition 7}

As in the proof of Proposition 6, it is sufficient to prove that $Q^{*}(0)$ can be sustained in some MPE.

Let us consider the following strategy profile.

1. Effort levels: let both agents choose an effort level optimal for a project of fixed scope $Q^{*}(0)$, and put zero effort for any state $q>Q^{*}(0)$.

2. Agenda setter proposals: let the agenda setter propose to stop the project for any state $q \geq Q^{*}(0)$, and continue to project for all $q<Q^{*}(0)$.

3. Agent $j$ 's decisions: let agent $j$ accept the agenda setter's proposal to stop for all states $q \geq Q^{*}(0)$, and otherwise accept to stop whenever $J(q ; q) \geq J\left(q ; Q^{*}(0)\right)$.

To show such strategy profile is an MPE, we must show that agents play a best response to each other at every state.

Let us fix the strategy of the agenda setter and check that agent $j$ 's strategy is a best response at every state.

- First, suppose agent 1 is the agenda setter. If agent 2 is offered to stop the project at a state $q \geq Q^{*}(0)$, he should accept: agent 1 puts no effort past state 
$Q^{*}(0)$, and agent 2 would rather not work alone on the project as $\widehat{Q}_{2}<Q^{*}(0)$. If agent 2 is offered to stop at a state $q<Q^{*}(0)$, he should accept only if the payoff he makes from immediate project termination, $J_{2}(q ; q)$ is no less than the payoff he makes by rejecting - which then pushes back the next anticipated proposal at state $Q^{*}(0), J_{2}\left(q ; Q^{*}(0)\right)$. Given the agenda setter's strategy, agent 2 expects to complete the project in state $Q^{*}(0)$, and by definition of agent 2's effort strategy, the effort levels of agent 2 are optimal at all states.

- Second, suppose agent 2 is the agenda setter. If agent 1 is offered to stop the project at $q \geq Q^{*}(0)$, then agent 1 finds it optimal to accept because agent 1 is never in favor of continuing the project past $\bar{Q}_{1}$. If agent 1 is offered to stop the project at $q<Q^{*}(0)$, then he should accept only if the payoff from immediate project termination $J_{1}(q ; q)$ is no less than the payoff he expects to make from rejecting, which as before is $J_{1}\left(q ; Q^{*}(0)\right)$. Given the agenda setter's strategy, agent 1 expects to complete the project in state $Q^{*}(0)$, and by definition of agent 1 's effort strategy, the effort levels of agent 1 are optimal at all states.

Next let us fix the strategy of agent $j$ and check that the agenda setter's strategy is a best response at every state.

- First, suppose agent 1 is the agenda setter. Then agent 1 expects to make payoff $J\left(q ; Q^{*}(0)\right)$ by following the conjectured equilibrium strategy. To make a better payoff, he would have to complete the project at a state $Q<Q^{*}(0)$. However such a proposal to stop the project early would not be accepted by agent 2, who is better off working towards a project of scope $Q^{*}(0)$. Hence not proposing to stop before state $Q^{*}(0)$ is a (weak) best response. As agent 2 accepts to stop at all states $q \geq Q^{*}(0)$ agent 1 is better off proposing to stop at all states $q \geq Q^{*}(0)$. Agent 1 anticipates the project scope to be $Q^{*}(0)$ and his effort levels are optimal for such a project scope.

- Second, suppose agent 2 is the agenda setter. Then agent 2 expects to make payoff $J\left(q ; Q^{*}(0)\right)$ by following the conjectured equilibrium strategy, and to make a larger payoff would require completing the project at a state $Q>Q^{*}(0)$. Therefore it is never optimal for agent 2 to stop before reaching the surplusmaximizing project scope. However it is always optimal to stop at every $Q \geq$ $Q^{*}(0)$, as agent 1 plans to put in no effort after $Q^{*}(0)$, and agent 2 prefers not to work alone on the project since $\widehat{Q}_{2}<Q^{*}(0)$. 
Hence the conjectured strategy profile constitutes a project-completing MPE with project scope $Q^{*}(0)$.

\section{A.11 Proof of Proposition 8}

Fix some $Q>0$. We use the normalization $\widetilde{J}_{i}(q)=\frac{J_{i}(q)}{\gamma_{i}}$ as in the proof of Proposition 1.

To prove part 1 , assume that $\frac{\gamma_{1}}{\alpha_{1}}<\frac{\gamma_{2}}{\alpha_{2}}$, let $\widetilde{D}(q)=\widetilde{J}_{1}(q)-\widetilde{J}_{2}(q)$, and note that $\widetilde{D}(\cdot)$ is smooth, $\lim _{q \rightarrow-\infty} \widetilde{D}(q)=0$ and $\widetilde{D}(Q)=\left(\frac{\alpha_{1}}{\gamma_{1}}-\frac{\alpha_{2}}{\gamma_{2}}\right) Q>0$. Suppose that $\widetilde{D}(\cdot)$ has an interior global extreme point, and denote such extreme point by $\bar{q}$. Because $\widetilde{D}(\cdot)$ is smooth, it must be the case that $\widetilde{D}^{\prime}(\bar{q})=0$. Then it follows from $(5)$ that $r \widetilde{D}(\bar{q})=\frac{\sigma^{2}}{2} \widetilde{D}^{\prime \prime}(\bar{q})$. If $\bar{q}$ is a maximum, then $\widetilde{D}^{\prime \prime}(\bar{q}) \leq 0$, so $\widetilde{D}(\bar{q}) \leq 0$, which contradicts the fact that $\lim _{q \rightarrow-\infty} \widetilde{D}(q)=0$ and the assumption that $\bar{q}$ is a maximum. On the other hand, if $\bar{q}$ is a minimum, then $\widetilde{D}^{\prime \prime}(\bar{q}) \geq 0$, so $\widetilde{D}(\bar{q}) \geq 0$, which contradicts the fact that $\lim _{q \rightarrow-\infty} \widetilde{D}(q)=0$ and the assumption that $\bar{q}$ is a minimum. Therefore, $\widetilde{D}^{\prime}(q)>0$ for all $q$, which implies that $a_{1}(q)>a_{2}(q)$ for all $q$.

To prove part 2 , let $D(q)=\frac{J_{1}(q)}{\alpha_{1}}-\frac{J_{2}(q)}{\alpha_{2}}$, and note that $D(\cdot)$ is smooth, $\lim _{q \rightarrow-\infty} D(q)=$ 0 , and $D(Q)=0$. Therefore, either $D(q)=0$ for all $q$, or $D(\cdot)$ has an interior global extreme point. Suppose that the former is true. Then for all $q$, we have $D(q)=D^{\prime}(q)=D^{\prime \prime}(q)=0$, which using (3) implies that

$$
r D(q)=\frac{\left[J_{1}^{\prime}(q)\right]^{2}}{2 \alpha_{1}^{2}}\left(\frac{\alpha_{2}}{\gamma_{2}}-\frac{\alpha_{1}}{\gamma_{1}}\right)=0 \Longrightarrow J_{1}^{\prime}(q)=0 .
$$

By Proposition 1, we have $J_{i}^{\prime}>0$ in any project-completing MPE so this is a contradiction. Thus the latter must be true. Then there exists some $\bar{q}$ such that $D^{\prime}(\bar{q})=0$. Using $(3)$, this implies that

$$
r D(\bar{q})=\frac{\left[J_{1}^{\prime}(\bar{q})\right]^{2}}{2 \alpha_{1}^{2}}\left(\frac{\alpha_{2}}{\gamma_{2}}-\frac{\alpha_{1}}{\gamma_{1}}\right)+\frac{\sigma^{2}}{2} D^{\prime \prime}(\bar{q}),
$$

and note that $J_{1}^{\prime}(\bar{q})>0$. Suppose that $\bar{q}$ is a maximum. Then $D^{\prime \prime}(\bar{q}) \leq 0$, which together with the fact that $\frac{\alpha_{2}}{\gamma_{2}}<\frac{\alpha_{1}}{\gamma_{1}}$ implies that $D(\bar{q})<0$. Therefore, $D(q) \leq 0$ for all $q$, which completes the proof of part 2 .

Finally, if $\frac{\alpha_{1}}{\gamma_{1}}=\frac{\alpha_{2}}{\gamma_{2}}$, then it follows from the analysis above that $\widetilde{D}(q)=\widetilde{D}^{\prime}(q)=0$ and $D(q)=0$, which implies that $a_{1}(q)=a_{2}(q)$ and $\frac{J_{1}(q)}{\alpha_{1}}=\frac{J_{2}(q)}{\alpha_{2}}$ for all $q \geq 0$. 


\section{References}

Acemoglu, D. and Robinson, J. A. (2006a), 'De Facto Political Power and Institutional Persistence', American Economic Review: Papers and Proceedings 96(2), 325-330.

Acemoglu, D. and Robinson, J. A. (2006b), Economic Origins of dictatorship and democracy, New York: Cambridge University Press.

Acemoglu, D. and Robinson, J. A. (2008), 'The Persistence of Power, Elites and Institutions', The American Economc Review 98(1), 267-293.

Admati, A. and Perry, M. (1991), 'Joint Projects without Commitment', Review of Economic Studies 58, 259-276.

Aghion, P. and Tirole, J. (1997), 'Formal and Real Authority in Organizations', The Journal of Political Economy 105(1), 1-29.

Akerlof, R. J. (2015), 'A Theory of Authority', Working paper.

Associated Press (2015), 'Contractors Sought for New Detroit-Windsor Bridge', The Detroit News July 20, 2015.

Atkinson, K., Han, W. and Stewart, D. (2009), Numerical Solution of Ordinary Differential Equations, John Wiley \& Sons, Inc., Hoboken, New Jersey.

Bagwell, K. and Staiger, R. W. (2002), The Economics of the World Trading System, MIT Press.

Baron, D. P. (1996), 'A Dynamic Theory of Collective Goods Programs', The American Political Science Review 90(2), 316-330.

Battaglini, M. and Coate, S. (2008), 'A Dynamic Theory of Public Spending, Taxation and Debt', American Economic Review 98(1), 201-236.

Battaglini, M. and Harstad, B. (forthcoming), 'Particiation and Duration of Environmental Agreements', Journal of Political Economy .

Battaglini, M., Nunnari, S. and Palfrey, T. (2014), 'Dynamic Free Riding with Irreversible Investments', American Economic Review 104(9), 2858-71.

Beshkar, M. and Bond, E. (2010), 'Flexibility in Trade Agreements', Working paper . 
Besley, T. and Persson, T. (2011), Pillars of Propsperity: The Political Economics of Development Clusters, Princeton: Princeton University Press.

Bhagwati, J. and Sutherland, P. (2011), The Doha Round: Setting a Deadline, Defining a Final Deal. Interim Report of the High Level Trade Experts Group. January.

Bonatti, A. and Rantakari, H. (forthcoming), 'The Politics of Compromise', American Economic Review .

Bowen, T. R. (2013), 'Forbearance in Optimal Multilateral Agreement', Working paper.

Bowen, T. R., Chen, Y. and Eraslan, H. (2014), 'Mandatory Versus Discretionary Spending: The Status Quo Effect', American Economic Review 104(10), 2941-2974.

Bureau of Labor Statistics (2011), 'International Comparison of Manufacturing Productivity and Unit Labor Cost Trends'.

Callander, S. (2008), 'A Theory of Policy Expertise', Quarterly Journal of Political Science 3, 123-140.

Callander, S. and Harstad, B. (2015), 'Experimentation in Federal Systems', Quarterly Journal of Economics 130(2), 951-1002.

Compte, O. and Jehiel, P. (2004), 'Gradualism in Bargaining and Contribution Games', The Review of Economic Studies 71(4), 975-1000.

Cvitanić, J. and Georgiadis, G. (2015), 'Achieving Efficiency in Dynamic Contribution Games', Working paper.

Diermeier, D. and Fong, P. (2011), 'Legislative Bargaining with Reconsideration', Quarterly Journal of Economics 126(2), 947-985.

Dixit, A., Grossman, G. M. and Gul, F. (2000), 'The Dynamics of Political Compromise', Journal of Political Economy 108(3), 531-568.

Dobson, M. (2004), The Triple Constraints in Project Management: Project Management Essential Library, Management Concepts Inc.,.

Fershtman, C. and Nitzan, S. (1991), 'Dynamic Voluntary Provision of Public Goods', European Economic Review 35, 1057-1067. 
Georgiadis, G. (2015), 'Project and Team Dynamics', Review of Economic Studies 82(1), 187-218.

Georgiadis, G., Lippman, S. and Tang, C. (2014), 'Project Design with Limited Commitment and Teams', RAND Journal of Economics 45(3), 598-623.

Hirsch, A. V. and Shotts, K. W. (2015), 'Competitive Policy Development', American Economic Review 105(4), 1646-1664.

Kamien, M. I. and Schwartz, N. L. (2012), Dynamic Optimization: The Calculus of Variations and Optimal Control in Economics and Management, Dover Publications, Inc. Mineloa, NY.

Kessing, S. G. (2007), 'Strategic Complementarity in the Dynamic Private Provision of a Discrete Public Good', Journal of Public Economic Theory 9(4), 699-710.

Levhari, D. and Mirman, L. J. (1980), 'The Great Fish War: An Example Using a Dynamic Cournot-Nash Solution', The Bell Journal of Economics 11(1), 322-334.

Lizzeri, A. and Persico, N. (2001), 'The Provision of Public Goods under Alternative Electoral Incentives', American Economic Review 91(1), 225-239.

Maggi, G. (2014), International Trade Agreements in The Handbook of International Economics, Vol. 4, Elsevier. eds Gita Gopinath and Elhanan Helpman and Kenneth Rogoff.

Marx, L. M. and Matthews, S. A. (2000), 'Dynamic Voluntary Contribution to a Public Project', Review of Economic Studies 67, 327-358.

Nordhaus, W. (2015), 'Climate Clubs: Overcoming Free-riding in International Climate Policy', American Economic Review 105(4), 1339-1370.

Pfeffer, J. (1981), Power in Organizations, Financial Times Prentice Hall.

Plumer, B. (2014), 'NASA wants to keep the International Space Station going until 2024. Is that a good idea?', The Washington Post January 9, 2014.

Romer, T. and Rosenthal, H. (1979), 'Bureaucrats Versus Voters: On the Political Economy of Resource Allocation by Direct Democracy', The Quarterly Journal of Economics 93(4), 563-587. 
Strulovici, B. (2010), 'Learning while Voting: Determinants of Collective Experimentation', Econometrica 78(3), 933-971.

Weber, M. (1958), 'The Three Types of Legitimate Rule', Berkeley Publications in Society and Institutions 4(1), 1-11.

Williamson, O. E. (1996), Efficiency, Power, Authority and Economic Organization, Kluwer Academic Publishers, eds John Groenewegen.

Yakovenko, A. (1999), 'The Intergovernmental Agreement on the International Space Station', Space Policy 15, 79-86.

Yamamoto, F. J., Miyairi, T., Regmi, M. B., Moon, J. R. and Cable, B. (2003), Asian Highway Handbook, United Nations.

Yildirim, H. (2006), 'Getting the Ball Rolling: Voluntary Contributions to a LargeScale Public Project', Journal of Public Economic Theory 8, 503-528. 